\title{
Linking Combinatorial and Classical Dynamics: Conley Index and Morse Decompositions
}

\author{
Bogdan Batko ${ }^{1} \cdot$ Tomasz Kaczynski $^{2} \cdot$ Marian Mrozek $^{1} \cdot$ Thomas Wanner $^{3}$
}

Received: 18 October 2017 / Revised: 15 August 2019 / Accepted: 4 November 2019 /

Published online: 23 January 2020

(c) The Author(s) 2020

\begin{abstract}
We prove that every combinatorial dynamical system in the sense of Forman, defined on a family of simplices of a simplicial complex, gives rise to a multivalued dynamical system $F$ on the geometric realization of the simplicial complex. Moreover, $F$ may be chosen in such a way that the isolated invariant sets, Conley indices, Morse decompositions and Conley-Morse graphs of the combinatorial vector field give rise to isomorphic objects in the multivalued map case.
\end{abstract}

Keywords Combinatorial vector field · Multivalued dynamical system - Simplicial complex · Discrete Morse theory · Conley theory $\cdot$ Morse decomposition ·

Conley-Morse graph · Isolated invariant set · Isolating block

Mathematics Subject Classification Primary: 37B30 - 54H20; Secondary: 37B35 . 37E15 $\cdot 57 \mathrm{M} 99 \cdot 57 \mathrm{Q} 05 \cdot 57 \mathrm{Q} 15$

\section{Introduction}

In the years since Forman $[14,15]$ introduced combinatorial vector fields on simplicial complexes, they have found numerous applications in such areas as visualization and mesh compression [21], graph braid groups [13], homology computation [17,25],

Communicated by Shmuel Weinberger.

Research of B.B. and M.M. was partially supported by the Polish National Science Center under Maestro Grant No. 2014/14/A/ST1/00453. Research of T.K. was supported by a Discovery Grant from NSERC of Canada. T.W. was partially supported by NSF Grants DMS-1114923 and DMS-1407087, and by the Simons Foundation under Award 581334. All authors gratefully acknowledge the support of Hausdorff Research Institute for Mathematics in Bonn for providing an excellent environment to work together during the 2017 Special Hausdorff Program on Applied and Computational Algebraic Topology.

$\bowtie \quad$ Marian Mrozek

Marian.Mrozek@uj.edu.pl

Extended author information available on the last page of the article 
astronomy [38], the study of Čech and Delaunay complexes [6], and many others. One reason for this success has its roots in Forman's original motivation. In his papers, he sought to transfer the rich dynamical theories due to Morse [26] and Conley [9] from the continuous setting of a continuum (connected compact metric space) to the finite, combinatorial setting of a simplicial complex. This has proved to be extremely useful for establishing finite, combinatorial results via ideas from dynamical systems. In particular, Forman's theory yields an alternative when studying sampled dynamical systems. The classical approach consists in the numerical study of the dynamics of the differential equation constructed from the sample. The construction uses the data in the sample either to discover the natural laws governing the dynamics [37] in order to write the equations or to interpolate or approximate directly the unknown vector field in the differential equations [7]. In the emerging alternative, one can eliminate differential equations and study directly the combinatorial dynamics defined by the sample [14,15,20,33,34].

The two approaches are essentially distinct. On the one hand, dynamical systems defined by differential equations on a differentiable manifold arise in a wide variety of applications and show an extreme wealth of observable dynamical behavior, at the expense of fairly involved mathematical techniques which are needed for their precise description. On the other hand, the discrete simplicial complex setting makes the study of many phenomena simple, due to the availability of fast combinatorial algorithms. This leads to the natural question of which approach should be chosen when for a given problem.

In order to answer this question, it may be helpful to go beyond the exchange of abstract underlying ideas present in much of the existing work and look for the precise relation between the two theories. In our previous paper [19], we took this path and studied the formal ties of multivalued dynamics in the combinatorial and continuum settings. The choice of multivalued dynamics is natural because the combinatorial vector fields generate multivalued dynamics in a natural way. Moreover, in the finite setting such dynamical phenomena as homoclinic or heteroclinic connections are not possible in single-valued dynamics. The choice of multivalued dynamics on continua is not a restriction. This is a broadly studied and well-understood theory. The theory originated in the middle of the twentieth century from the study of contingent equations and differential inclusions [3,36,42] and control theory [35]. At the end of the twentieth century, it was successfully applied to computer-assisted proofs in dynamics [24,32]. In particular, the Conley theory for multivalued dynamics was studied by several authors $[4,5,11,12,18,28,40]$.

In [19] we proved that for any combinatorial vector field on the collection of simplices of a simplicial complex, one can construct an acyclic-valued and upper semicontinuous map on the underlying geometric realization whose dynamics on the level of invariant sets exhibits the same complexity. More precisely, by introducing the notion of isolated invariant sets in the discrete setting, we could show that every isolated invariant set of the combinatorial vector field gives rise to a corresponding isolated invariant set in the classical multivalued setting. We also presented a link between the combinatorial and the classical multivalued dynamics on the level of individual dynamical trajectories.

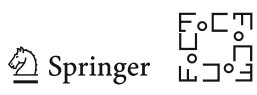




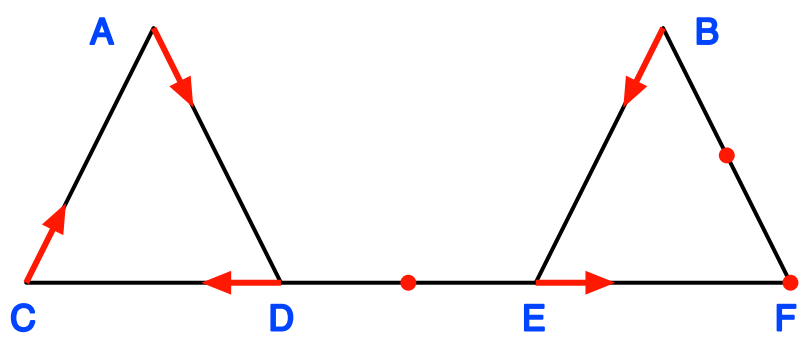

Fig. 1 Sample discrete vector field. This figure shows a simplicial complex $\mathcal{X}$ which is a graph on six vertices with seven edges. Critical cells are indicated by red dots; vectors of the vector field are shown as red arrows (Color figure online)

In the present paper, we complete the program started in [19] by showing that the above-described correspondence extends to Conley indices of the corresponding isolated invariant set as well as Morse decompositions and Conley-Morse graphs [2,8], a global descriptor of dynamics capturing its gradient structure.

The organization of the paper is as follows: In Sect. 2 we present the main result of the paper and illustrate it with some examples. In Sect. 3 we recall the basics of the Conley theory for multivalued dynamics. In Sect. 4 we recall from [19] the construction of a multivalued self-map $F: X \multimap X$ associated with a combinatorial vector field $\mathcal{V}$ on a simplicial complex $\mathcal{X}$ with the geometric realization $X:=|\mathcal{X}|$. In Sect. 5 we use this construction to outline the proof of the main result of the paper in a series of auxiliary theorems. The remaining sections are devoted to the proofs of these theorems.

\section{Main Result}

Let $\mathcal{X}$ denote the family of simplices of a finite abstract simplicial complex. The face relation on $\mathcal{X}$ defines on $\mathcal{X}$ the $T_{0}$ Alexandrov topology [1]. A subset $\mathcal{A} \subseteq \mathcal{X}$ is open in this topology if all cofaces of any element of $\mathcal{A}$ are also in $\mathcal{A}$. The closure of $\mathcal{A}$ in this topology, denoted $\mathrm{Cl} \mathcal{A}$, is the family of all faces of all simplices in $\mathcal{A}$ (see Sect. 3.1 for more details). A combinatorial vector field $\mathcal{V}$ on $\mathcal{X}$ is a partition of $\mathcal{X}$ into non-empty subsets of cardinality at most two such that each subset of cardinality two (a doubleton) consists of a simplex $\sigma$ and a coface of $\sigma$ of codimension one. A simplex belonging to a singleton in $\mathcal{V}$ is referred to as a critical cell. A doubleton in $\mathcal{V}$ ordered into a pair with the lower-dimensional simplex coming first is referred to as a vector. In the sequel, in order to simplify the language, we identify a singleton with the critical cell it contains and a doubleton with the associated vector.

The elementary example in Fig. 1 presents a one-dimensional simplicial complex $\mathcal{X}$ consisting of six vertices $\{A, B, C, D, E, F\}$ and seven edges $\{A C, A D, B E$, $B F, C D, D E, E F\}$, and the combinatorial vector field consisting of three singletons (critical cells) $\{\{B F\},\{D E\},\{F\}\}$ and five doubletons (vectors) $\{\{A, A D\},\{B, B E\}$, $\{C, A C\},\{D, C D\},\{E, E F\}\}$. With a combinatorial vector field $\mathcal{V}$, we associate multivalued dynamics given as iterates of a multivalued map $\Pi_{\mathcal{V}}: \mathcal{X} \multimap \mathcal{X}$ sending each critical simplex to all of its faces, each source of a vector to the corresponding target, and each target of a vector to all faces of the target other than the corresponding source 


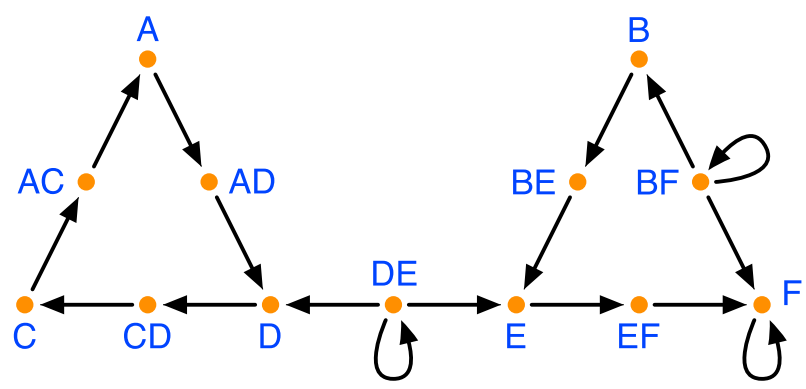

Fig. 2 The directed graph $G_{\mathcal{V}}$ for the combinatorial vector field in Fig. 1

and the target itself. In the case of the example in Fig. 1, the map is (we skip the braces in the case of singletons to keep the notation simple)

$$
\begin{aligned}
\Pi_{\mathcal{V}}=\{ & (A, A D),(A D, D),(B, B E),(B E, E),(B F,\{B, B F, F\}), \\
& (C, A C),(A C, A),(C D, C)(D, C D),(D E,\{D, D E, E\}), \\
& (E, E F),(E F, F),(F, F)\} .
\end{aligned}
$$

The multivalued map $\Pi_{\mathcal{V}}$ may be considered as a directed graph $G \mathcal{V}$ with vertices in $\mathcal{X}$ and an arrow from a simplex $\sigma$ to a simplex $\tau$ whenever $\tau \in \Pi_{\mathcal{V}}(\sigma)$. The directed graph $G \mathcal{V}$ for the combinatorial vector field in Fig. 1 is presented in Fig. 2. A subset $\mathcal{A} \subseteq \mathcal{X}$ is invariant with respect to $\mathcal{V}$ if every element of $\mathcal{A}$ is both a head and a tail of an arrow in $G_{\mathcal{V}}$ which joins vertices in $\mathcal{A}$. An element $\sigma \in \mathrm{Cl} \mathcal{A} \backslash \mathcal{A}$ is an internal tangency of $\mathcal{A}$ if it admits both an arrow originating in $\sigma$ with its head in $\mathcal{A}$ and an arrow terminating in $\sigma$ with its tail in $\mathcal{A}$. The set $\operatorname{Ex} \mathcal{A}:=\mathrm{Cl} \mathcal{A} \backslash \mathcal{A}$ is referred to as the exit set of $\mathcal{A}$ (see [19, Definition 3.4]) or mouth of $\mathcal{A}$ (see [33, Sect. 4.4]). An invariant $\mathcal{S}$ set is an isolated invariant set if the exit set Ex $\mathcal{S}$ is closed and it admits no internal tangencies. While the notion of internal tangency might seem strange at first sight, it is motivated by the classical situation in which isolated invariant sets can be enclosed in the interior of isolating blocks - and the later ones cannot exhibit any internal tangencies. In the combinatorial setting, there is not enough space to carry over these definitions precisely, but our use of the term serves the same purpose.

Note that $\mathcal{X}$ itself is an isolated invariant set if and only if it is invariant. The (co)homological Conley index of an isolated invariant set $\mathcal{S}$ is the relative singular (co)homology of the pair $(\mathrm{Cl} \mathcal{S}, \mathrm{Ex} \mathcal{S})$ with topology induced from the Alexandrov topology in $X$. Note that $(\mathrm{Cl} \mathcal{S}, \mathrm{Ex} \mathcal{S})$ is a pair of simplicial subcomplexes of the simplicial complex $\mathcal{X}$. Therefore, by McCord's Theorem [22], the singular (co)homology of the pair $(\mathrm{Cl} \mathcal{S}, \operatorname{Ex} \mathcal{S})$ is isomorphic to the simplicial homology of the pair $(\mathrm{Cl} \mathcal{S}, \operatorname{Ex} \mathcal{S})$.

The singleton $\{B F\}$ in Fig. 1 is an example of an isolated invariant set of $\mathcal{V}$. Its exit set is $\{B, F\}$, and its Conley index is the (co)homology of the pointed circle. Another example is the set $\{A, A C, A D, C, C D, D\}$ with an empty exit set and the Conley index equal to the (co)homology of the circle. Both these examples are minimal isolated invariant sets, that is, none of their proper non-empty subsets is an isolated invariant set.

The two-dimensional example depicted in Fig. 3 presents a simplicial complex which is built from 10 triangles, 19 edges and 10 vertices, and a combinato-

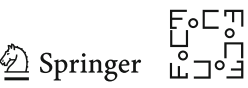




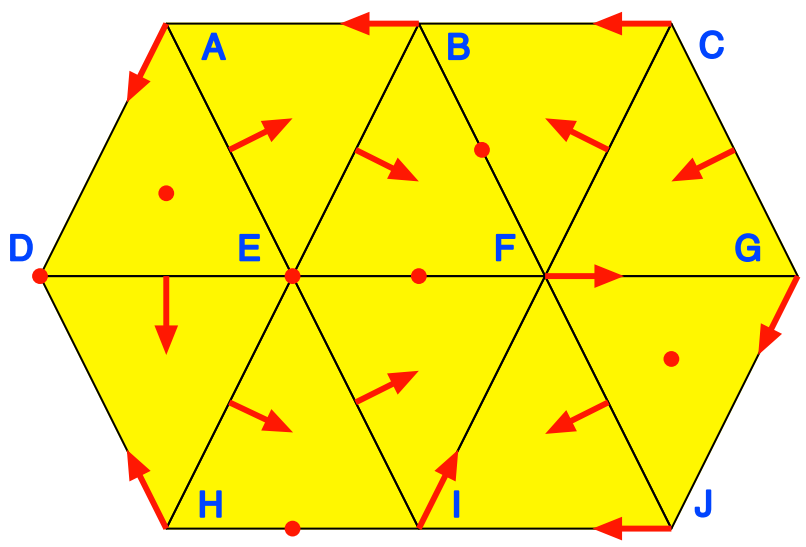

Fig. 3 Sample discrete vector field. This figure shows a simplicial complex $\mathcal{X}$ which triangulates a hexagon (shown in yellow), together with a discrete vector field. Critical cells are indicated by red dots; vectors of the vector field are shown as red arrows. This example will be discussed throughout the paper (Color figure online)

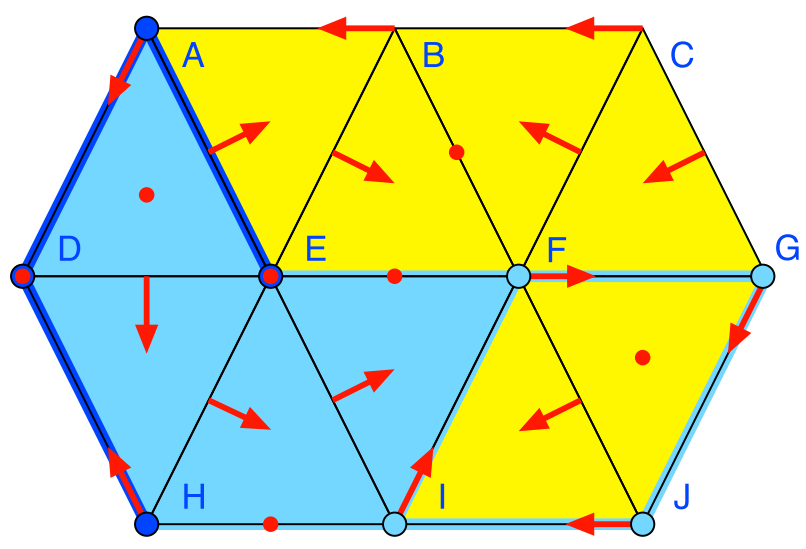

Fig. 4 Sample isolated invariant set for the discrete vector field shown in Fig. 3. The simplices which belong to the isolated invariant set $\mathcal{S}$ are indicated in light blue and are given by four vertices, nine edges, and four triangles. Its exit set Ex $\mathcal{S}$ is shown in dark blue, and it consists of four vertices and three edges (Color figure online)

rial vector field consisting of 7 critical cells and a total of 16 vectors. The set $\{A D E, D E, D E H, E F, E F I, E H, E H I, E I, F, F G, F I, G, G J, H I, I, I J, J\}$ is an example of an isolated invariant set for this combinatorial vector field. It is presented in Fig. 4. Its exit set is $\{A, A D, A E, D, D H, E, H\}$, and its Conley index is the (co)homology of the pointed circle. This isolated invariant set is not minimal. For instance, the singleton $\{E F\}$ is a subset which itself is an isolated invariant set.

A connection from an isolated invariant set $\mathcal{S}_{1}$ to an isolated invariant set $\mathcal{S}_{2}$ is a sequence of vertices on a walk in $G \mathcal{V}$ originating in $\mathcal{S}_{1}$ and terminating in $\mathcal{S}_{2}$. A family $\mathcal{M}=\left\{\mathcal{M}_{p} \mid p \in \mathbb{P}\right\}$ indexed by a poset $\mathbb{P}$ and consisting of mutually disjoint isolated invariant subsets of an isolated invariant set $\mathcal{S}$ is a Morse decomposition of $\mathcal{S}$ if any connection between elements in $\mathcal{M}$ which is not contained entirely in one of 

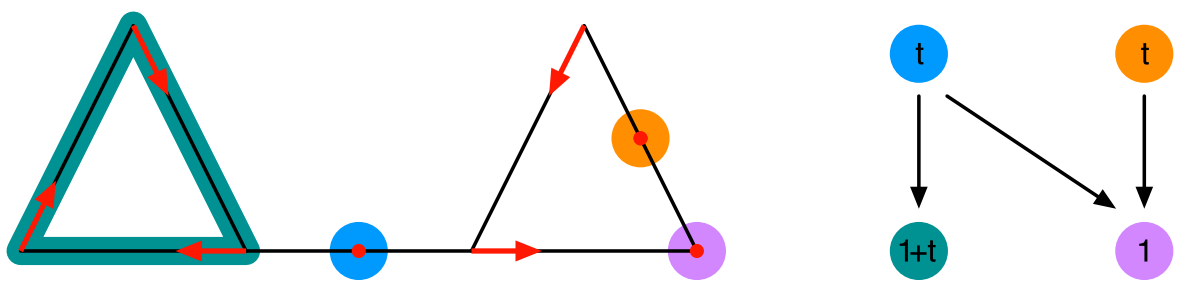

Fig. 5 Morse decomposition for the example shown in Fig. 1. For this example, one can find four minimal Morse sets, which are indicated in the left image in different colors. The right image shows the associated Conley-Morse graph. The labels inside vertices are the Poincaré polynomials of the respective Conley indices (Color figure online)
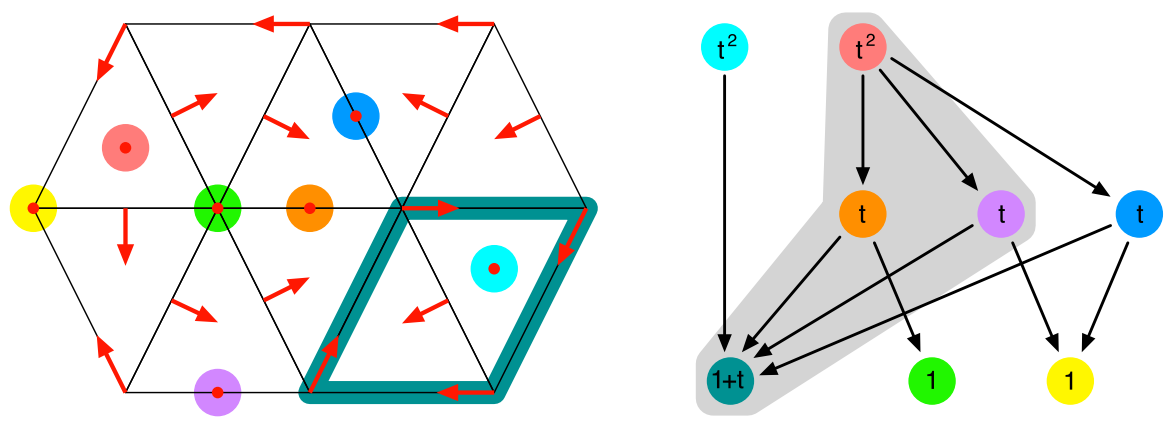

Fig. 6 Morse decomposition for the example shown in Fig. 3. For this example, one can find eight minimal Morse sets, which are indicated in the left image in different colors. The right image shows the associated Conely-Morse graph. The labels inside vertices are the Poincaré polynomials of the respective Conley indices. The isolated invariant set shown in Fig. 4 corresponds to the subgraph indicated by the gray shaded area in the Morse graph (Color figure online)

the elements of $\mathcal{M}$ originates in $\mathcal{M}_{q^{\prime}}$ and terminates in $\mathcal{M}_{q}$ with $q^{\prime}>q$. The isolated invariant sets in $\mathcal{M}$ are referred to as Morse sets. The associated Conley-Morse graph is the partial order induced on $\mathcal{M}$ by the existence of connections and represented as a directed graph labeled with the Conley indices of the isolated invariant sets in $\mathcal{M}$. Typically, the labels are written as Poincaré polynomials, that is, polynomials whose $i$ th coefficient equals the $i$ th Betti number of the Conley index.

An example of a Morse decomposition for the combinatorial vector field in Fig. 1 is

$$
\mathcal{M}:=\{\{B F\},\{F\},\{D E\},\{A, A D, C, C A, C D, D\}\} .
$$

The respective Poincaré polynomials of Conley indices in this example are: $t, 1, t$ and $1+t$. The corresponding Conley-Morse graph is presented in Fig. 5. A Morse decomposition of the example in Fig. 3 together with the associated Conley-Morse graph is presented in Fig. 6.

The main result of this paper is the following theorem.

Theorem 2.1 For every combinatorial vector field $\mathcal{V}$ on a simplicial complex $\mathcal{X}$, there exists an upper semicontinuous acyclic multivalued map $F:|\mathcal{X}| \multimap|\mathcal{X}|$ on the geometric realization $|\mathcal{X}|$ of $\mathcal{X}$, which induces the identity in homology, such that

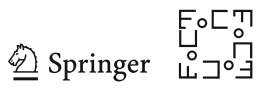




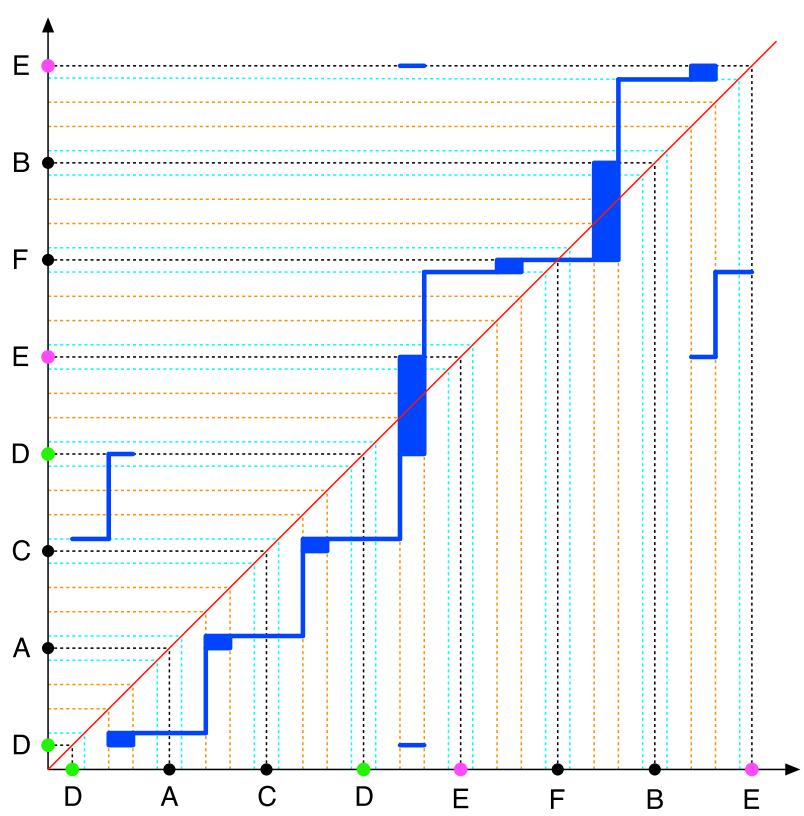

Fig. 7 The multivalued map $F$ for the combinatorial vector field shown in Fig. 1. For visualization purposes, the domain of $F$ is straightened to a segment in which vertices $D$ (marked in green) and $E$ (marked in magenta) are represented twice. The graph of $F$ is shown in blue. The edge $D E$ in the middle corresponds to the center edge in Fig. 1. To its left, the three line segments correspond to the cycle in the combinatorial vector field. Note that the two green vertices are identified. The three edges to the right of the center correspond to the right triangle in Fig. 1. Also here the two magenta vertices are identified (Color figure online)

(i) for every Morse decomposition $\mathcal{M}$ of $\mathcal{V}$, there exists a Morse decomposition $M$ of the semidynamical system induced by $F$,

(ii) the Conley-Morse graph of $M$ is isomorphic to the Conley-Morse graph of $\mathcal{M}$,

(iii) each element of $M$ is contained in the geometric representation of the corresponding element of $\mathcal{M}$.

This theorem is an immediate consequence of the more detailed theorems presented in Sect. 5. The multivalued map $F$ constructed in the proof for Theorem 2.1, for the example in Fig. 1, is presented in Fig. 7.

\section{Conley Theory for Multivalued Topological Dynamics}

In this section, we recall the main concepts of Conley theory for multivalued dynamics in the combinatorial and classical setting: isolated invariant sets, index pairs, Conley index and Morse decompositions. The construction of the Conley index for multivalued maps on locally compact metric spaces $[5,18]$ is based on the Alexander-Spanier cohomology because the strong excision property (cf. [39, Chapter 6.6]) and the Vietoris-Begle theorem (cf. [39, Chapter 6.9]) for this theory are readily available. A recent, not published, observation is that an analogous construction of a homological 
Conley theory for multivalued dynamics in the classical setting is also possible. Such a construction is achieved by replacing Alexander-Spanier cohomology with a homology theory for which both the strong excision property and the Vietoris-Begle theorem hold, for instance Steenrod homology (cf. [10,23]). The results of this paper are also valid for such a homology Conley index. We emphasize this by writing (co)homology instead of cohomology throughout the paper.

The choice of a coefficient ring for (co)homology does not matter in the Conley index construction. In applications usually field coefficients suffice. Such a choice is also convenient in the selection of a normal functor, another algebraic tool needed in the construction of the Conley index for maps. This is because a very elementary normal functor, namely the Leray functor, may be used in vector spaces (see Sect. 3.3). Thus, to keep the presentation simple, we assume the coefficient ring is a field.

\subsection{Preliminaries}

We write $f: X \nrightarrow Y$ to denote a partial function, that is, a function whose domain, denoted $\operatorname{dom} f$, is a subset of $X$. We write im $f:=f(X)$ to denote the image of $f$ and Fix $f:=\{x \in \operatorname{dom} f \mid f(x)=x\}$ to denote the set of fixed points of $f$.

Given a topological space $X$ and a subset $A \subseteq X$, we denote by $\mathrm{cl} A$, int $A$ and bd $A$, respectively, the closure, the interior and the boundary of $A$. We often use the set ex $A:=\operatorname{cl} A \backslash A$ which we call the exit set or mouth of $A$. Whenever applying an operator like cl or ex to a singleton, we drop the braces to keep the notation simple.

The singular cohomology (homology) of the pair $(X, A)$ is denoted by $H^{*}(X, A)$ (respectively, $H_{*}(X, A)$ ). Note that in this paper, we apply (co)homology only to polyhedral pairs or pairs weakly homotopy equivalent to polyhedral pairs. Hence, the singular (co)homology is the same as Alexander-Spanier cohomology (Steenrod homology). In particular, all but a finite number of Betti numbers of the pair $(X, A)$ are zero. The corresponding Poincaré polynomial is the polynomial whose $i$ th coefficient is the $i$ th Betti number.

By a multivalued map $F: X \multimap X$, we mean a map from $X$ to the family of nonempty subsets of $X$. We say that $F$ is upper semicontinuous if for any open $U \subseteq X$, the set $\{x \in X \mid F(x) \subseteq U\}$ is open. We say that $F$ is strongly upper semicontinuous if for any $x \in X$, there exists a neighborhood $U$ of $X$ such that $x^{\prime} \in U$ implies $F\left(x^{\prime}\right) \subseteq F(x)$. Note that every strongly upper semicontinuous multivalued map is upper semicontinuous. We say that $F$ is acyclic-valued if $F(x)$ is acyclic for any $x \in X$.

We consider a simplicial complex as a finite family $\mathcal{X}$ of finite sets such that any non-empty subset of a set in $\mathcal{X}$ is in $\mathcal{X}$. We refer to the elements of $\mathcal{X}$ as simplices. By the dimension of a simplex, we mean one less than its cardinality. We denote by $\mathcal{X}_{k}$ the set of simplices of dimension $k$. A vertex is a simplex of dimension zero. If $\sigma, \tau \in \mathcal{X}$ are simplices and $\tau \subseteq \sigma$, then we say that $\tau$ is a face of $\sigma$ and $\sigma$ is a coface of $\tau$. An $(n-1)$-dimensional face of an $n$-dimensional simplex is called a facet. We say that a subset $\mathcal{A} \subseteq \mathcal{X}$ is open if all cofaces of any element of $\mathcal{A}$ are also in $\mathcal{A}$. It is easy to see that the family of all open sets of $\mathcal{X}$ is a $T_{0}$ topology on $\mathcal{X}$, called Alexandrov topology. It corresponds to the face poset of $\mathcal{X}$ via the Alexandrov theorem [1]. In

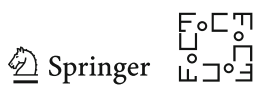


particular, the closure of $\mathcal{A} \subseteq \mathcal{X}$ in the Alexandrov topology consists of all faces of simplices in $\mathcal{A}$. To avoid confusion, in the case of the Alexandrov topology we write $\mathrm{Cl} \mathcal{A}$ and Ex $\mathcal{A}$ for the closure and the exit of $\mathcal{A} \subseteq \mathcal{X}$.

By identifying vertices of an $n$-dimensional simplex $\sigma$ with a closed convex hull of $n+1$ linearly independent vectors in $\mathbb{R}^{d}$ with $d>n$, we obtain a geometric realization of $\sigma$. We denote it by $|\sigma|$. However, whenever the meaning is clear from the context, we drop the bars to keep the notation simple. By choosing the identification in such a way that all vectors corresponding to vertices of $\mathcal{X}$ are linearly independent, we obtain a geometric realization of $\mathcal{X}$ given by

$$
|\mathcal{X}|:=\bigcup_{\sigma \in \mathcal{X}}|\sigma|
$$

Note that up to a homeomorphism, the geometric realization does not depend on a particular choice of the identification. In the sequel we assume that a simplicial complex $\mathcal{X}$ and its geometric realization $X:=|\mathcal{X}|$ are fixed. Given a vertex $v \in \mathcal{X}_{0}$, we denote by $t_{v}:|\mathcal{X}| \rightarrow[0,1]$ the map which assigns to each point $x \in|\mathcal{X}|$ its barycentric coordinate with respect to the vertex $v$ whenever $x$ belongs to a simplex in the star of $v$ and 0 otherwise. For a simplex $\sigma \in \mathcal{X}$ the open cell of $\sigma$ is

$$
\stackrel{\circ}{\sigma}:=\left\{x \in|\sigma| \mid t_{v}(x)>0 \text { for } v \in \sigma\right\} .
$$

For $\mathcal{A} \subseteq \mathcal{X}$ we write

$$
\langle\mathcal{A}\rangle:=\bigcup_{\sigma \in \mathcal{A}} \stackrel{\circ}{\sigma} .
$$

One easily verifies the following proposition.

Proposition 3.1 We have the following properties

(i) if $\mathcal{A}$ is closed in $\mathcal{X}$, then $|\mathcal{A}|=\langle\mathcal{A}\rangle$,

(ii) if $\operatorname{Ex} \mathcal{A}$ is closed in $\mathcal{X}$, then $\langle\mathcal{A}\rangle=|\mathcal{A}| \backslash|\operatorname{Ex} \mathcal{A}|$.

\subsection{Combinatorial Case}

The concept of a combinatorial vector field was introduced by Forman [15]. There are a few equivalent ways of stating its definition. The definition introduced in Sect. 2 is among the simplest: A combinatorial vector field on a simplicial complex $\mathcal{X}$ is a partition $\mathcal{V}$ of $\mathcal{X}$ into singletons and doubletons such that each doubleton consists of a simplex and one of its facets. The partition induces an injective partial map which sends the element of each singleton to itself and each facet in a doubleton to its coface in the same doubleton. This leads to the following equivalent definition which will be used in the rest of the paper. 
Definition 3.2 (see [19, Definition 3.1]) An injective partial self-map $\mathcal{V}: \mathcal{X} \nrightarrow \mathcal{X}$ of a simplicial complex $\mathcal{X}$ is called a combinatorial vector field, or also a discrete vector field if

(i) For every simplex $\sigma \in \operatorname{dom} \mathcal{V}$ either $\mathcal{V}(\sigma)=\sigma$, or $\sigma$ is a facet of $\mathcal{V}(\sigma)$.

(ii) $\operatorname{dom} \mathcal{V} \cup \operatorname{im} \mathcal{V}=\mathcal{X}$,

(iii) $\operatorname{dom} \mathcal{V} \cap \operatorname{im} \mathcal{V}=\operatorname{Fix} \mathcal{V}$

Note that every combinatorial vector field is a special case of a combinatorial multivector field introduced and studied in [33].

Given a combinatorial vector field $\mathcal{V}$ on $\mathcal{X}$, we define the associated combinatorial multivalued flow as the multivalued map $\Pi_{\mathcal{V}}: \mathcal{X} \multimap \mathcal{X}$ given by

$$
\Pi_{\mathcal{V}}(\sigma):= \begin{cases}\mathrm{Cl} \sigma & \text { if } \sigma \in \operatorname{Fix} \mathcal{V} \\ \operatorname{Ex} \sigma \backslash\left\{\mathcal{V}^{-1}(\sigma)\right\} & \text { if } \sigma \in \operatorname{im} \mathcal{V} \backslash \operatorname{Fix}(\mathcal{V}), \\ \{\mathcal{V}(\sigma)\} & \text { if } \sigma \in \operatorname{dom} \mathcal{V} \backslash \operatorname{Fix}(\mathcal{V})\end{cases}
$$

For the rest of the paper, we assume that $\mathcal{V}$ is a fixed combinatorial vector field on $\mathcal{X}$ and $\Pi_{\mathcal{V}}$ denotes the associated combinatorial multivalued flow.

A solution of the flow $\Pi_{\mathcal{V}}$ is a partial function $\varrho: \mathbb{Z} \nrightarrow \mathcal{X}$ such that $\varrho(i+1) \in$ $\Pi_{\mathcal{V}}(\varrho(i))$ whenever $i, i+1 \in \operatorname{dom} \varrho$. The solution $\varrho$ is full if $\operatorname{dom} \varrho=\mathbb{Z}$. The invariant part of $\mathcal{S} \subseteq \mathcal{X}$, denoted Inv $\mathcal{S}$, is the collection of those simplices $\sigma \in \mathcal{S}$ for which there exists a full solution $\varrho: \mathbb{Z} \rightarrow \mathcal{S}$ such that $\varrho(0)=\sigma$. A set $\mathcal{S} \subseteq \mathcal{X}$ is invariant if $\operatorname{Inv} \mathcal{S}=\mathcal{S}$.

Definition 3.3 (see [19, Definition 3.4]) A subset $\mathcal{S} \subseteq \mathcal{X}$, invariant with respect to a combinatorial vector field $\mathcal{V}$, is called an isolated invariant set if the exit set $\operatorname{Ex} \mathcal{S}=$ $\mathrm{Cl} \mathcal{S} \backslash \mathcal{S}$ is closed and there is no solution $\varrho:\{-1,0,1\} \rightarrow \mathcal{X}$ such that $\varrho(-1), \varrho(1) \in$ $\mathcal{S}$ and $\varrho(0) \in \operatorname{Ex} \mathcal{S}$. The closure $\mathrm{Cl} \mathcal{S}$ is called an isolating block for the isolated invariant set $\mathcal{S}$.

Proposition 3.4 (see [19, Proposition 3.7]) An invariant set $\mathcal{S} \subseteq \mathcal{X}$ is an isolated invariant set if $\operatorname{Ex} \mathcal{S}$ is closed and for every $\sigma \in \mathcal{X}$ we have $\sigma^{-} \in \mathcal{S}$ if and only if $\sigma^{+} \in \mathcal{S}$, where

$$
\sigma^{+}:=\left\{\begin{array}{ll}
\mathcal{V}(\sigma) & \text { if } \sigma \in \operatorname{dom} \mathcal{V} \\
\sigma & \text { otherwise }
\end{array} \quad \text { and } \quad \sigma^{-}:= \begin{cases}\sigma & \text { if } \sigma \in \operatorname{dom} \mathcal{V} \\
\mathcal{V}^{-1}(\sigma) & \text { otherwise }\end{cases}\right.
$$

An immediate consequence of Proposition 3.4 is the following corollary.

Corollary 3.5 If $\mathcal{S}$ is an isolated invariant set, then for any $\tau \in \mathcal{X}$ and $\sigma, \sigma^{\prime} \in \mathcal{S}$ we have

$$
\sigma \subseteq \tau \subseteq \sigma^{\prime} \Longrightarrow \tau \in \mathcal{S}
$$

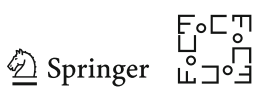


A pair $\mathcal{P}=\left(\mathcal{P}_{1}, \mathcal{P}_{2}\right)$ of closed subsets of $\mathcal{X}$ such that $\mathcal{P}_{2} \subseteq \mathcal{P}_{1}$ is an index pair for $\mathcal{S}$ if the following three conditions are satisfied

$$
\begin{aligned}
& \mathcal{P}_{1} \cap \Pi_{\mathcal{V}}\left(\mathcal{P}_{2}\right) \subseteq \mathcal{P}_{2}, \\
& \Pi_{\mathcal{V}}\left(\mathcal{P}_{1} \backslash \mathcal{P}_{2}\right) \subseteq \mathcal{P}_{1}, \\
& \mathcal{S}=\operatorname{Inv}\left(\mathcal{P}_{1} \backslash \mathcal{P}_{2}\right) .
\end{aligned}
$$

By [33, Theorem 7.11] the pair $(\mathrm{Cl} \mathcal{S}, \mathrm{Ex} \mathcal{S})$ is an index pair for $\mathcal{S}$ and the (co)homology of the index pair of $\mathcal{S}$ does not depend on the particular choice of index pair but only on $\mathcal{S}$. Hence, by definition, it is the Conley index of $\mathcal{S}$. We denote it $\operatorname{Con}(\mathcal{S})$.

\subsection{Classical Case}

The study of the Conley index for multivalued maps was initiated in [18] with a restrictive concept of the isolating neighborhood, limiting possible applications. In particular, that theory is not satisfactory for the needs of this paper. These limitations were removed by a new theory developed recently in $[4,5]$. We recall the main concepts of the generalized theory below.

Let $F: X \multimap X$ be an upper semicontinuous map with compact, acyclic values. A partial map $\varrho: \mathbb{Z} \nrightarrow X$ is called a solution for $F$ through $x \in X$ if we have both $\varrho(0)=x$ and $\varrho(n+1) \in F(\varrho(n))$ for all $n, n+1 \in \operatorname{dom} \varrho$. Given $N \subseteq X$, we define its invariant part by

$$
\operatorname{Inv} N:=\{x \in N \mid \exists \varrho: \mathbb{Z} \rightarrow N \text { which is a solution for } F \text { through } x\}
$$

A compact set $N \subseteq X$ is an isolating neighborhood for $F$ if Inv $N \subseteq$ int $N$. The $F$-boundary of a given set $A \subseteq X$ is

$$
\operatorname{bd}_{F}(A):=\operatorname{cl} A \cap \operatorname{cl}(F(A) \backslash A) .
$$

Definition 3.6 A pair $P=\left(P_{1}, P_{2}\right)$ of compact sets $P_{2} \subseteq P_{1} \subseteq N$ is a weak index pair for $F$ in $N$ if the following properties are satisfied.

(a) $F\left(P_{i}\right) \cap N \subseteq P_{i}$ for $i=1,2$,

(b) $\operatorname{bd}_{F}\left(P_{1}\right) \subseteq P_{2}$,

(c) $\operatorname{Inv} N \subseteq \operatorname{int}\left(P_{1} \backslash P_{2}\right)$,

(d) $P_{1} \backslash P_{2} \subseteq$ int $N$.

Unlike the Conley index for flows and the Conley index for combinatorial vector fields, the (co)homology of a weak index pair is not an invariant of Inv $N$. To extract common information from two index pairs, the following construction is needed. For the weak index pair $P$, we set

$$
T(P):=T_{N}(P):=\left(P_{1} \cup(X \backslash \text { int } N), P_{2} \cup(X \backslash \text { int } N)\right) .
$$


and define the associated index map $I_{P}$ as the composition $H^{*}\left(F_{P}\right) \circ H^{*}\left(i_{P}\right)^{-1}$ $\left(H^{*}\left(i_{P}\right)^{-1} \circ H^{*}\left(F_{P}\right)\right.$ in the case of homology), where $F_{P}: P \multimap T(P)$ is the restriction of $F$ and $i_{P}: P \multimap T(P)$ is the inclusion map. The pair $\left(H^{*}(P), I_{P}\right)$ is an example of an object in the category of endomorphisms $\operatorname{Endo}(\mathcal{E})$ defined as follows. Given a category $\mathcal{E}$, objects in $\operatorname{Endo}(\mathcal{E})$ are pairs $(E, e)$ with $E$ an object in $\mathcal{E}$ and $e: E \rightarrow E$ a morphism in $E$. A morphism $\varphi:(E, e) \rightarrow\left(E^{\prime}, e^{\prime}\right)$ in $\operatorname{Endo}(\mathcal{E})$ is a morphism $\varphi: E \rightarrow E$ in $\mathcal{E}$ such that $\varphi e=e^{\prime} \varphi$. To extract information from $\left(H^{*}(P), I_{P}\right)$ which is an invariant of Inv $N$, one needs a normal functor $N$ : $\operatorname{Endo}(\mathcal{E}) \rightarrow \mathcal{E}^{\prime}$ that is a functor which sends the endomorphism $e$ considered as a morphism $e:(E, e) \rightarrow(E, e)$ in $\operatorname{Endo}(\mathcal{E})$ to an isomorphism in $\mathcal{E}^{\prime}$. The existence of a universal normal functor for any category $\mathcal{E}$ is guaranteed by a result of A. Szymczak [41]. In practice, one chooses a normal functor which is easy to compute. An example of such a normal functor is the Leray functor $(\operatorname{see}[27]) L: \operatorname{Endo}(\mathcal{E}) \rightarrow \operatorname{Auto}(\mathcal{E})$ where $\operatorname{Auto}(\mathcal{E})$ is the full subcategory of $\operatorname{Endo}(\mathcal{E})$ whose objects have the form $(E, e)$ where $e$ is an isomorphism in $\mathcal{E}$. We refer to $L(E, e)$ as the Leray reduction of $(E, e)$. For the needs of this paper, it suffices to describe the Leray reduction for the special case of the category Vect $f$ of finite-dimensional vector spaces. In this case $L(E, e):=$ ( $E /$ gker $e, e^{\prime}$ ) where gker $e=\sum_{n=1}^{\infty} \operatorname{ker} e^{n}$ and $e^{\prime}$ is the map induced on equivalence classes. For more details concerning normal functors and their use in the construction of the Conley index, we refer the reader to [30,31].

The Leray functor lets us to define the (co)homological Conley index of an isolated invariant set $S$ as $\operatorname{Con}(S, F):=L\left(H^{*}(P), I_{P}\right)$. The correctness of the definition is the consequence of the following two results.

Theorem 3.7 (see [5, Theorem 4.12]) For every neighborhood $W$ of $\operatorname{Inv} N$, there exists a weak index pair $P$ in $N$ such that $P_{1} \backslash P_{2} \subseteq W$.

Theorem 3.8 (see [5, Theorem 6.4]) The module $L\left(H^{*}(P), I_{P}\right)$ is independent of the choice of an isolating neighborhood $N$ for $S$ and of a weak index pair $P$ in $N$.

It is worth to note that Leray functor or another normal functor is needed only in the construction of the Conley index for dynamical systems with discrete time (iterates of maps). The reason is that unlike the translations along the trajectories of a flow, a general map need not be homotopic to identity. If a map is homotopic to identity, then one can prove that the map induced on $H^{*}(P) / \operatorname{gker} I_{P}$ is the identity and in consequence the Leray reduction of $\left(H^{*}(P), I_{P}\right)$ may be identified with $H^{*}(P)$ (cf. [29]). As we prove in Theorem 5.3, this is the case for the multivalued map constructed in this paper. And, this is what one would expect because the multivalued map we construct is modeled on a combinatorial analogue of a classical vector field giving rise to flow-type dynamics.

\subsection{Morse Decompositions}

In order to formulate the definition of the Morse decomposition of an isolated invariant set, we need the concepts of $\alpha$ - and $\omega$-limit sets. We formulate these definitions independently in the combinatorial and classical settings. Given a full solution $\varrho: \mathbb{Z} \rightarrow \mathcal{X}$

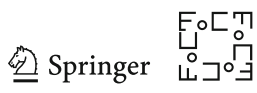


of the combinatorial dynamics $\Pi_{\mathcal{V}}$ on $\mathcal{X}$, the $\alpha$ - and $\omega$-limit sets of $\varrho$ are, respectively, the sets

$$
\alpha(\varrho):=\bigcap_{k \in \mathbb{Z}}\{\varrho(n) \mid n \leq k\}, \quad \omega(\varrho):=\bigcap_{k \in \mathbb{Z}}\{\varrho(n) \mid n \geq k\} .
$$

Note that $\alpha$-and $\omega$-limit sets of $\Pi_{\mathcal{V}}$ are always non-empty invariant sets because $\mathcal{X}$ is finite.

Now, given a solution $\varphi: \mathbb{Z} \rightarrow X$ of a multivalued upper semicontinuous map $F: X \multimap X$, we define its $\alpha$ - and $\omega$-limit sets, respectively, by

$$
\alpha(\varphi):=\bigcap_{k \in \mathbb{Z}} \operatorname{cl} \varphi((-\infty, k]) \quad \omega(\varphi):=\bigcap_{k \in \mathbb{Z}} \operatorname{cl} \varphi([k,+\infty)) .
$$

Definition 3.9 Let $\mathcal{S}$ be an isolated invariant set of $\Pi_{\mathcal{V}}: \mathcal{X} \multimap \mathcal{X}$. We say that the family $\mathcal{M}:=\left\{\mathcal{M}_{r} \mid r \in \mathbb{P}\right\}$ indexed by a poset $\mathbb{P}$ is a Morse decomposition of $\mathcal{S}$ if the following conditions are satisfied:

(a) the elements of $\mathcal{M}$ are mutually disjoint isolated invariant subsets of $\mathcal{S}$,

(b) for every full solution $\varphi$ in $X$, there exist $r, r^{\prime} \in \mathbb{P}, r \leq r^{\prime}$, such that $\alpha(\varphi) \subseteq \mathcal{M}_{r^{\prime}}$ and $\omega(\varphi) \subseteq \mathcal{M}_{r}$,

(c) if for a full solution $\varphi$ in $\mathcal{X}$ and $r \in \mathbb{P}$ we have $\alpha(\varphi) \cup \omega(\varphi) \subseteq \mathcal{M}_{r}$, then $\operatorname{im} \varphi \subseteq \mathcal{M}_{r}$.

By replacing in the above definition the combinatorial multivalued map $\Pi_{\mathcal{V}}$ on $\mathcal{X}$ by an upper semicontinuous map $F: X \rightarrow X$ and adjusting the notation accordingly, we obtain the definition of the Morse decomposition $M:=\left\{M_{r} \mid r \in \mathbb{P}\right\}$ of an isolated invariant set $S$ of $F$.

It is not difficult to observe that Definition 3.9 in the combinatorial setting is equivalent to the brief definition of Morse decomposition given in terms of connections in Sect. 2. Moreover, in the case of combinatorial vector fields, Definition 3.9 coincides with the definition presented in [33, Sect. 9.1].

\section{From Combinatorial to Classical Dynamics}

In this section, given a combinatorial vector field $\mathcal{V}$ on a simplicial complex $\mathcal{X}$, we recall from [19] the construction of a multivalued self-map $F=F_{\mathcal{V}}: X \multimap X$ on the geometric realization $X:=|\mathcal{X}|$ of $\mathcal{X}$. This map will be used to establish the correspondence of Conley indices, Morse decompositions and Conley-Morse graphs between the combinatorial and classical multivalued dynamics as outlined in the introduction.

\subsection{Cellular Decomposition}

We begin by presenting a special cellular complex representation of $X=|\mathcal{X}|$ used in the construction of the multivalued map $F$. For this we need some terminology. 
Let $d$ denote the maximal dimension of the simplices in $\mathcal{X}$. Fix a $\lambda \in \mathbb{R}$ such that $0 \leq \lambda<\frac{1}{d+1}$ and a point $x \in X$. The $\lambda$-signature of $x$ is the function

$$
\operatorname{sign}^{\lambda} x: \mathcal{X}_{0} \ni v \mapsto \operatorname{sgn}\left(t_{v}(x)-\lambda\right) \in\{-1,0,1\},
$$

where sgn $: \mathbb{R} \rightarrow\{-1,0,1\}$ is the standard sign function. Then, a simplex $\sigma \in \mathcal{X}$ is a $\lambda$-characteristic simplex of $x$ if both $\left.\operatorname{sign}^{\lambda} x\right|_{\sigma} \geq 0$ and $\left(\operatorname{sign}^{\lambda} x\right)^{-1}(\{1\}) \subseteq \sigma$ are satisfied. We denote the family of $\lambda$-characteristic simplices of $x$ by

$$
\mathcal{X}^{\lambda}(x):=\left\{\sigma \in \mathcal{X} \mid\left(\operatorname{sign}^{\lambda} x\right)^{-1}(\{1\}) \subseteq \sigma \text { and } \operatorname{sign}^{\lambda} x(v) \geq 0 \text { for all } v \in \sigma\right\}
$$

For any $\lambda \geq 0$, the set $\left(\operatorname{sign}^{\lambda} x\right)^{-1}(\{1\})$ is a simplex. We call it the minimal $\lambda$ characteristic simplex of $x$, and we denote it by $\sigma_{\min }^{\lambda}(x)$. Note that

$$
\sigma=\sigma_{\min }^{0}(x) \Longleftrightarrow x \in \stackrel{\circ}{\sigma}
$$

If $\lambda>0$, then the set $\left(\operatorname{sign}^{\lambda} x\right)^{-1}(\{0,1\})$ is also a simplex. We call it the maximal $\lambda$-characteristic simplex of $x$, and we denote it by $\sigma_{\max }^{\lambda}(x)$.

Lemma 4.1 (see [19, Lemma 4.2]) If $0 \leq \varepsilon<\lambda<\frac{1}{1+d}$, then $\sigma_{\max }^{\lambda}(x) \subseteq \sigma_{\text {min }}^{\varepsilon}$ (x) for any $x \in X=|\mathcal{X}|$.

Lemma 4.2 (see [19, Lemma 4.3]) For all $x \in X=|\mathcal{X}|$ we have $\mathcal{X}^{\lambda}(x) \neq \varnothing$. Moreover, there exists a neighborhood $U$ of the point $x$ such that $\mathcal{X}^{\lambda}(y) \subseteq \mathcal{X}^{\lambda}(x)$ for all $y \in U$.

Given a triple $\left(\sigma_{1}, \sigma_{2}, \sigma\right)$ such that $\sigma_{1} \subseteq \sigma_{2} \subseteq \sigma \in \mathcal{X}$ by a $\lambda$-cell generated by this triple, we mean

$$
\left\langle\sigma_{1}, \sigma_{2}, \sigma\right\rangle_{\lambda}:=\left\{x \in X \mid \sigma_{\min }^{\lambda}(x)=\sigma_{1}, \sigma_{\max }^{\lambda}(x)=\sigma_{2}, \sigma_{\min }^{0}(x)=\sigma\right\} .
$$

Similarly, as in [19, Formulas (12) and (13)]), one can prove the following characterizations of $\left\langle\sigma_{1}, \sigma_{2}, \sigma\right\rangle_{\lambda}$ and its closure in terms of barycentric coordinates:

$$
\begin{aligned}
& \left\langle\sigma_{1}, \sigma_{2}, \sigma\right\rangle_{\lambda}=\left\{x \in X \mid t_{v}(x)>\lambda \text { for all } v \in \sigma_{1}\right. \text { and } \\
& t_{v}(x)<\lambda \text { for all } v \notin \sigma_{2} \text { and } \\
& \left.t_{v}(x)>0 \text { for all } v \in \sigma\right\}, \\
& \operatorname{cl}\left\langle\sigma_{1}, \sigma_{2}, \sigma\right\rangle_{\lambda}=\left\{x \in X \mid t_{v}(x) \geq \lambda \text { for all } v \in \sigma_{1}\right. \text { and } \\
& t_{v}(x) \leq \lambda \text { for all } v \notin \sigma_{2} \text { and } \\
& \left.t_{v}(x) \geq 0 \text { for all } v \in \sigma\right\} \text {. }
\end{aligned}
$$

It follows that $\left\langle\sigma_{1}, \sigma_{2}, \sigma\right\rangle_{\lambda}$ and its closure are convex in the affine subspace spanned by the vertices of $\sigma$ and $\left\{\left\langle\sigma_{1}, \sigma_{2}, \sigma\right\rangle_{\lambda} \mid \sigma_{1} \subseteq \sigma_{2} \subseteq \sigma \in \mathcal{X}\right\}$ forms a cellular decomposition of $X=|\mathcal{X}|$. We refer to it as the $\lambda$-cell decomposition of $X$.

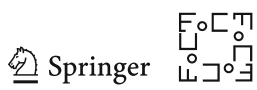


In the construction of the multivalued self-map $F=F_{\mathcal{V}}: X \multimap X$, a special role is played by

$$
\langle\sigma\rangle_{\lambda}:=\left\{x \in X \mid \mathcal{X}^{\lambda}(x)=\{\sigma\}\right\}=\sum_{\tau \in \mathcal{X}, \tau \supset \sigma}\langle\sigma, \sigma, \tau\rangle_{\lambda} .
$$

We refer to $\langle\sigma\rangle_{\lambda}$ as the $\lambda$-cell generated by $\sigma$. Directly from (8) and (9), we obtain the following characterizations of $\langle\sigma\rangle_{\lambda}$ and its closure in terms of barycentric coordinates:

$$
\begin{array}{r}
\langle\sigma\rangle_{\lambda}=\left\{x \in X \mid t_{v}(x)>\lambda \text { for all } v \in \sigma\right. \text { and } \\
\operatorname{cl}\left(t_{v}(x)<\lambda \text { for all } v \notin \sigma\right\}, \\
\operatorname{cl}\langle\sigma\rangle_{\lambda}=\left\{x \in X \mid t_{v}(x) \geq \lambda \text { for all } v \in \sigma\right. \text { and } \\
\left.t_{v}(x) \leq \lambda \text { for all } v \notin \sigma\right\} .
\end{array}
$$

Then, the following proposition follows easily from (10).

Proposition 4.3 For $\lambda$ satisfying $0<\lambda<\frac{1}{d+1}$, the $\lambda$-cells are open in $|\mathcal{X}|$ and mutually disjoint.

Another characterization of $\operatorname{cl}\langle\sigma\rangle_{\lambda}$ is given by the following corollary.

Corollary 4.4 (see [19, Corollary 4.6]) The following statements are equivalent:

(i) $\sigma \in \mathcal{X}^{\lambda}(x)$,

(ii) $\sigma_{\min }^{\lambda}(x) \subseteq \sigma \subseteq \sigma_{\max }^{\lambda}(x)$,

(iii) $x \in \operatorname{cl}\langle\sigma\rangle_{\lambda}$.

Note that for a simplicial complex $\mathcal{X}$, we have the following easy to verify formula:

$$
|\mathcal{X}|=\bigcup_{\sigma \in \mathcal{X}} \operatorname{cl}\langle\sigma\rangle_{\lambda}
$$

The cells $\langle\sigma\rangle_{\lambda}$ for various values of $\lambda$ are visualized in Fig. 8. They are the building blocks for the multivalued map $F$.

\subsection{The Maps $F_{\sigma}$ and the Map $F$}

We now recall from [19] the construction of the strongly upper semicontinuous map $F$ associated with a combinatorial vector field. For this, we fix two constants

$$
0<\gamma<\varepsilon<\frac{1}{d+1}
$$

and for any $\sigma \in \mathcal{X}$, we define the closed sets

$$
\begin{aligned}
& A_{\sigma}:=\left\{x \in \sigma^{+} \mid t_{v}(x) \geq \gamma \text { for all } v \in \sigma^{-}\right\} \cup \sigma^{-}, \\
& B_{\sigma}:=\left\{x \in \sigma^{+} \mid \text {there exists a } v \in \sigma^{-} \text {with } t_{v}(x) \leq \gamma\right\},
\end{aligned}
$$




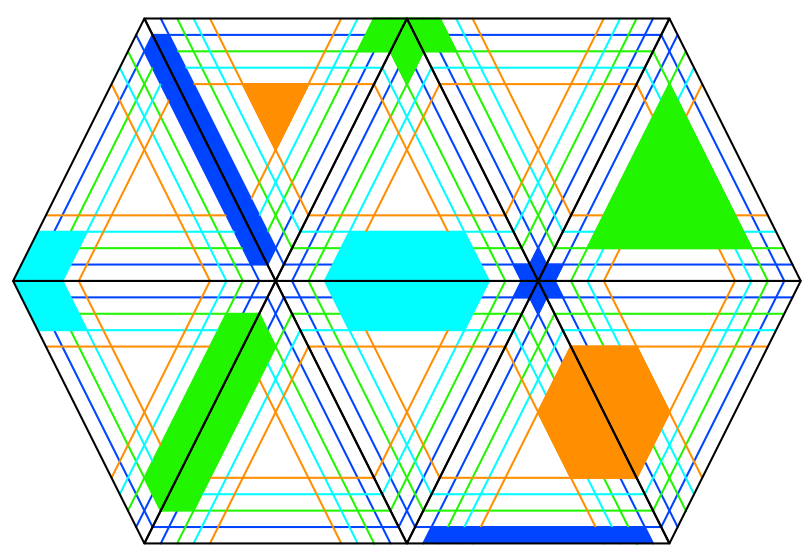

Fig. 8 Sample cell decomposition boundaries for the simplicial complex $\mathcal{X}$ from Fig. 3. The colored lines indicate the boundaries of $\varepsilon$-cells (orange), $\gamma$-cells (cyan), $\delta$-cells (green) and $\delta^{\prime}$-cells (blue). Throughout the paper, we assume that $0<\delta^{\prime}<\delta<\gamma<\varepsilon$. The figure also contains ten sample cells: Two orange $\varepsilon$-cells which are associated with a 2-simplex (upper left) and a 1-simplex (lower right); two cyan $\gamma$-cells which correspond to a 1-simplex (middle) and a 0 -simplex (left); three green $\delta$-cells for a 2 -simplex (upper right), a 1-simplex (lower left), and a 0 -simplex (top middle); as well as three blue $\delta^{\prime}$-cells for two 1-simplices (upper left and bottom right) and a 0 -simplex (right middle). All of these cells are open subsets of $|\mathcal{X}|$ (Color figure online)

$$
C_{\sigma}:=A_{\sigma} \cap B_{\sigma}
$$

Then, the following lemma is an immediate consequence of [19, Lemma 4.8].

Lemma 4.5 For any simplex $\sigma \in \mathcal{X} \backslash$ Fix $\mathcal{V}$, the sets $A_{\sigma}, B_{\sigma}$ and $C_{\sigma}$ are contractible.

For every simplex $\sigma \in \mathcal{X}$, we define a multivalued map $F_{\sigma}: X \multimap X$ by

$$
F_{\sigma}(x):= \begin{cases}\varnothing \quad \text { if } \sigma \notin \mathcal{X}^{\varepsilon}(x), \\ A_{\sigma} \quad \text { if } \sigma \in \mathcal{X}^{\varepsilon}(x), \sigma \neq \sigma_{\max }^{\varepsilon}(x)^{+}, \text {and } \sigma \neq \sigma_{\max }^{\varepsilon}(x)^{-}, \\ B_{\sigma} \quad \text { if } \sigma=\sigma_{\max }^{\varepsilon}(x)^{+} \neq \sigma_{\max }^{\varepsilon}(x)^{-}, \\ C_{\sigma} \quad \text { if } \sigma=\sigma_{\max }^{\varepsilon}(x)^{-} \neq \sigma_{\max }^{\varepsilon}(x)^{+}, \\ \sigma \quad \text { if } \sigma=\sigma_{\max }^{\varepsilon}(x)^{-}=\sigma_{\max }^{\varepsilon}(x)^{+},\end{cases}
$$

and the multivalued map $F: X \multimap X$ by

$$
F(x):=\bigcup_{\sigma \in \mathcal{X}} F_{\sigma}(x) \quad \text { for all } \quad x \in X=|\mathcal{X}| .
$$

Figure 7 shows the graph $\{(x, y) \in X \times X \mid y \in F(x)\}$ of the so-constructed map $F$ for the vector field in Fig. 1.

One of main results proved in [19] is the following theorem.

Theorem 4.6 (see [19, Theorem 4.12]) The map $F$ is strongly upper semicontinuous, and for every $x \in X$, the set $F(x)$ is non-empty and contractible.

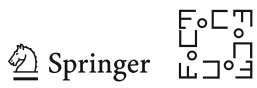




\section{The Correspondence Between Combinatorial and Classical Dynamics}

In this section we present the constructions and theorems establishing the correspondence between the multivalued dynamics of a combinatorial vector field $\mathcal{V}$ on the simplicial complex $\mathcal{X}$ and the associated multivalued dynamics of the multivalued map $F=F_{\mathcal{V}}$ constructed in Sect. 4. The theorems presented in this section provide the proof of Theorem 2.1.

Throughout the section we assume that $d$ is the maximal dimension of the simplices in $\mathcal{X}$.

\subsection{Correspondence of Isolated Invariant Sets}

In order to establish the correspondence on the level of isolated invariant sets, we fix a constant $\delta$ satisfying

$$
0<\delta<\gamma<\varepsilon<\frac{1}{d+1}
$$

where $\gamma$ and $\varepsilon$ are the constants chosen in Sect. 4.2 (see (13)). For $\mathcal{A} \subseteq \mathcal{X}$ and any constant $\beta$ satisfying $0<\beta<\frac{1}{d+1}$, we further set

$$
N_{\beta}(\mathcal{A}):=\bigcup_{\sigma \in \mathcal{A}} \operatorname{cl}\langle\sigma\rangle_{\beta} .
$$

Let $\mathcal{S} \subseteq \mathcal{X}$ be an isolated invariant set for the combinatorial vector field $\mathcal{V}$ in the sense of Definition 3.3. The following theorem associates with $\mathcal{S}$ an isolating block for $F$, and it was proved in [19].

Theorem 5.1 (see [19, Theorem 5.7]) The set

$$
N:=N_{\delta}:=N_{\delta}(\mathcal{S})
$$

is an isolating block for $F$. In particular, it is an isolating neighborhood for $F$.

A sample of an isolating block for the map $F$ given by (16) which corresponds to the combinatorial isolated invariant set in Fig. 4 is presented in Fig. 9.

Theorem 5.1 lets us to associate with $\mathcal{S}$ an isolated invariant set

$$
S(\mathcal{S}):=\operatorname{Inv} N_{\delta}
$$

given as the invariant part of $N_{\delta}$ with respect to $F$.

\subsection{The Conley Index of $S(\mathcal{S})$}

In order to compare the Conley indices of $\mathcal{S}$ and $S(\mathcal{S})$, we need to construct a weak index pair for $F$ in $N_{\delta}$. To define such a weak index pair, we choose another constant $\delta^{\prime}$ such that 


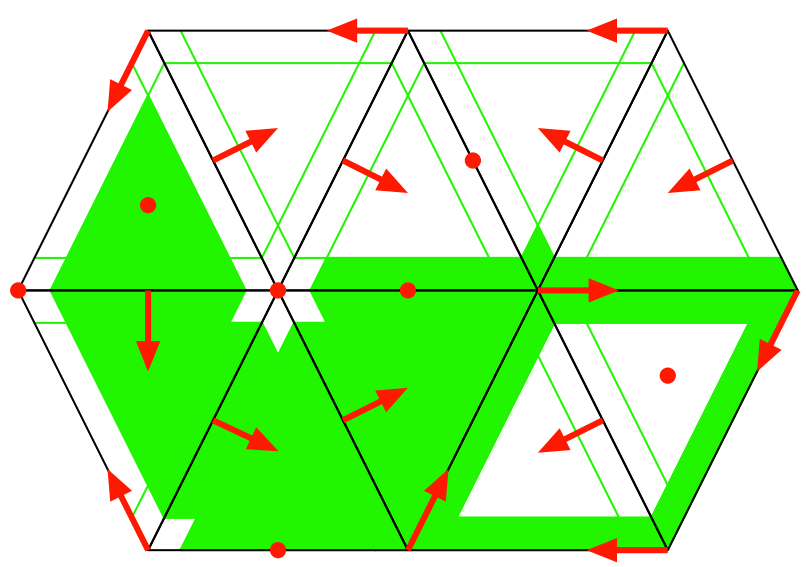

Fig. 9 Isolating block $N_{\delta}$ for the isolated invariant set $\mathcal{S}$ shown in Fig. 4. Notice that the block is the union of closed $\delta$-cells. For reference, we also show the $\delta$-cell boundaries outside $N_{\delta}$, but these are not part of the isolating block. The block is homeomorphic to a closed annulus

$$
0<\delta^{\prime}<\delta<\gamma<\varepsilon<\frac{1}{d+1},
$$

and for the remainder of this paper, these four constants will remain fixed. Now define the two sets

$$
P_{1}:=N_{\delta} \cap N_{\delta^{\prime}} \quad \text { and } \quad P_{2}:=N_{\delta^{\prime}} \cap \text { bd } N_{\delta} .
$$

Clearly, $P_{2} \subseteq P_{1} \subseteq N:=N_{\delta}$ are compact sets. We have the following theorem.

Theorem 5.2 The pair $P=\left(P_{1}, P_{2}\right)$ defined by (20) is a weak index pair for $F$ and the isolating neighborhood $N=N_{\delta}$.

The proof of Theorem 5.2 will be presented in Sect. 6. A weak index pair for the isolating block given in Fig. 9 is presented in Fig. 10. As recalled in Sect. 3.3, the Conley index of $S(\mathcal{S})$ with respect to $F$ is

$$
\operatorname{Con}(S(\mathcal{S}), F):=L\left(H^{*}(P), I_{P}\right)
$$

where $L$ is the Leray reduction of the relative (co)homology graded module $H^{*}(P)=$ $H^{*}\left(P_{1}, P_{2}\right)$ of $P$, and $I_{P}$ is the index map on $H^{*}(P)$. In Sect. 7 we prove the following theorem.

Theorem 5.3 We have

$$
\operatorname{Con}(S(\mathcal{S}), F) \cong\left(H^{*}(P), \operatorname{id}_{H^{*}(P)}\right)
$$

where $\mathrm{id}_{H^{*}(P)}$ denotes the identity map. In other words, as in the case offlows, the Conley index of $S(\mathcal{S})$ with respect to $F$ can be simply defined as the relative (co)homology $H^{*}(P)$.

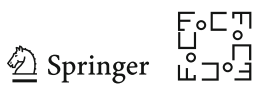




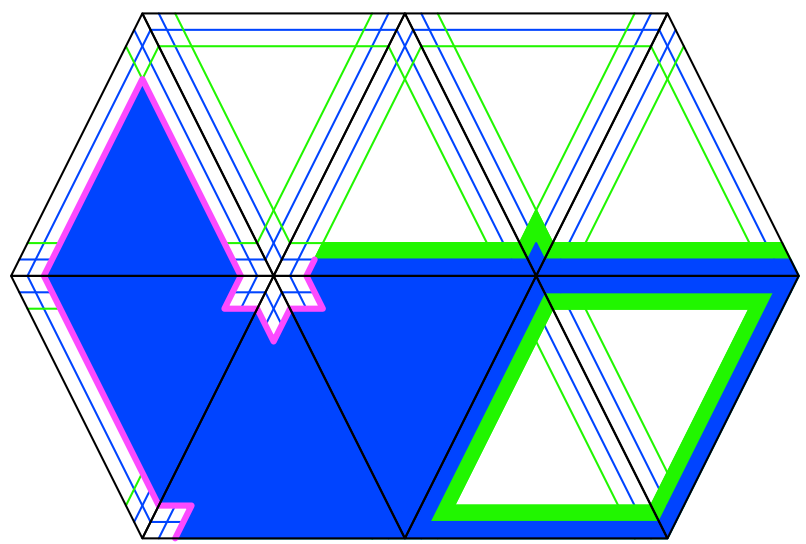

Fig. 10 The weak index pair $P=\left(P_{1}, P_{2}\right)$ associated with the isolating block $N_{\delta}$ from Fig. 9. The set $P_{1}$ is shown in dark blue, and the part of its boundary which comprises $P_{2}$ is indicated in magenta. Notice that the parts of the $\delta$-cells shown in green are cut from the isolating block $N_{\delta}$ when passing to $P_{1}$ (Color figure online)

\subsection{Correspondence of Conley Indices}

As recalled in Sect. 3.2, the Conley index of $\mathcal{S}$ with respect to $\Pi_{\mathcal{V}}$ is

$$
\operatorname{Con}(\mathcal{S}):=H^{*}(\mathrm{Cl} \mathcal{S}, \operatorname{Ex} \mathcal{S})
$$

In Sect. 8 we prove the following theorem.

Theorem 5.4 We have

$$
H^{*}\left(P_{1}, P_{2}\right) \cong H^{*}(\mathrm{Cl} \mathcal{S}, \operatorname{Ex} \mathcal{S})
$$

As a consequence,

$$
\operatorname{Con}(S(\mathcal{S})) \cong \operatorname{Con}(\mathcal{S})
$$

Theorem 5.4 extends the correspondence between the isolated invariant sets $\mathcal{S}$ and $S(\mathcal{S})$ to the respective Conley indices.

\subsection{Correspondence of Morse Decompositions}

Given $\mathcal{M}=\left\{\mathcal{M}_{r} \mid r \in \mathbb{P}\right\}$, a Morse decomposition of $\mathcal{X}$ with respect to the combinatorial flow $\Pi_{\mathcal{V}}$, we define the sets

$$
M_{r}:=N_{\varepsilon}^{r} \cap\left\langle\mathcal{M}_{r}\right\rangle,
$$


where $N_{\varepsilon}^{r}:=N_{\varepsilon}\left(\mathcal{M}_{r}\right)$ is given by (17), that is, we have

$$
N_{\varepsilon}^{r}=\bigcup_{\sigma \in \mathcal{M}_{r}} \operatorname{cl}\langle\sigma\rangle_{\varepsilon}
$$

In Sect. 9 we prove the following theorem, which establishes the correspondence between Morse decompositions of $\mathcal{V}$ and of $F$.

Theorem 5.5 The collection $M:=\left\{M_{r} \mid r \in \mathbb{P}\right\}$ is a Morse decomposition of $X$ with respect to $F$. Moreover, for each $r \in \mathbb{P}$ we have

$$
\operatorname{Con}\left(\mathcal{M}_{r}\right)=C\left(M_{r}\right)
$$

and the Conley-Morse graphs for the Morse decompositions $\mathcal{M}$ and $M$ coincide.

The reader can immediately see that Theorem 2.1, the main result of the paper, is now a consequence of Theorems 5.4 and 5.5.

\section{Proof of Theorem 5.2}

In this section we prove Theorem 5.2. The proof is split into six auxiliary lemmas and the verification that the pair $P$ defined by (20) satisfies the conditions (a) through (d) of Definition 3.6. Throughout this section, we make use of the constants chosen in (19).

\subsection{Auxiliary Lemmas}

Lemma 6.1 Consider a $\zeta$ satisfying $0<\zeta<\gamma$ and assume $A_{\sigma} \cap \mathrm{cl}\langle\tau\rangle_{\zeta} \neq \varnothing$ for all simplices $\tau, \sigma \in \mathcal{X}$. Then, either $\tau$ is a face of $\sigma^{-}$or $\tau=\sigma^{+}$.

Proof Choose an $x \in A_{\sigma} \cap \operatorname{cl}\langle\tau\rangle_{\zeta}$. According to (14), we have

$$
A_{\sigma}=\left\{\tilde{x} \in \sigma^{+} \mid t_{v}(\tilde{x}) \geq \gamma \text { for all } v \in \sigma^{-}\right\} \cup \sigma^{-} \text {. }
$$

If $\tau$ is a face of $\sigma^{-}$, we are done. Suppose that this does not hold. Then, $\tau$ has to contain the vertex $w$ of $\sigma^{+}$complementing $\sigma^{-}$as shown in Fig. 11 and $x \notin \sigma^{-}$. This implies that $t_{v}(x) \geq \gamma>\zeta$ for all $v \in \sigma^{-}$. Since

$$
\operatorname{cl}\langle\tau\rangle_{\zeta}=\left\{\tilde{x} \in X \mid t_{v}(\tilde{x}) \geq \zeta \text { for all } v \in \tau \text { and } t_{v}(x) \leq \zeta \text { for all } v \notin \tau\right\}
$$

this implies that all vertices of $\sigma^{-}$have to be in $\tau$. Hence, $\tau=\sigma^{+}$and the claim is proved.

Lemma 6.2 Suppose that $x \in \operatorname{cl}\langle\tau\rangle_{\delta}$ for some $\tau \in \mathcal{S}$ and that $\sigma:=\sigma_{\text {max }}^{\varepsilon}(x) \notin \mathcal{S}$. Then, for every $\zeta$ satisfying $0<\zeta<\gamma$, we have

$$
F(x) \cap N_{\zeta}=\varnothing .
$$

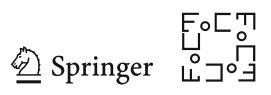


Fig. 11 The sets $\sigma^{+}, A_{\sigma}$ and vertex $w$ in the proof of Lemma 6.1

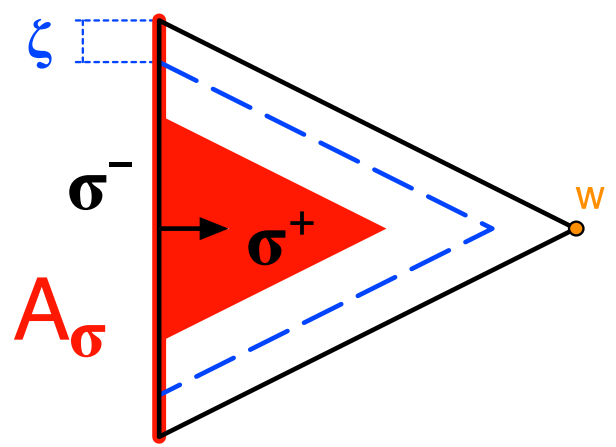

Proof Suppose that $F(x) \cap N_{\zeta} \neq \varnothing$. Hence, there exists a simplex $\hat{\tau} \in \mathcal{S}$ and a point $y \in F(x) \cap \operatorname{cl}\langle\hat{\tau}\rangle_{\zeta}$. Then, Lemma 4.1 and Corollary 4.4 imply that

$$
\sigma=\sigma_{\max }^{\varepsilon}(x) \subseteq \sigma_{\min }^{\delta}(x) \subseteq \tau
$$

In other words, $\sigma$ is a face of $\tau$. Since $\mathcal{S}$ is an isolated invariant set, $\tau \in \mathcal{S}$ implies the inclusions $\tau^{ \pm} \in \mathcal{S}$.

Since $y \in F(x)$, we have $y \in F_{\varrho}(x)$ for some simplex $\varrho \in \mathcal{X}^{\varepsilon}(x)$. There are four possible cases to consider.

First, assume that $\varrho \neq \sigma^{+}$and $\varrho \neq \sigma^{-}$. Then, $F_{\varrho}(x)=A_{\varrho}$ and $y \in A_{\varrho} \cap \operatorname{cl}\langle\hat{\tau}\rangle_{\zeta}$. According to Lemma 6.1, $\hat{\tau}$ has to be a face of $\varrho^{-}$or $\hat{\tau}=\varrho^{+}$. Recall that we assumed $\sigma \notin \mathcal{S}$. Since $\varrho \in \mathcal{X}^{\varepsilon}(x)$, we get

$$
\varrho \subseteq \sigma \subseteq \tau
$$

Since $\sigma \notin \mathcal{S}$ and $\tau \in \mathcal{S}$, Corollary 3.5 implies that $\varrho \notin \mathcal{S}$ and Proposition 3.4 implies that $\varrho^{ \pm} \notin \mathcal{S}$. Since we assumed $\hat{\tau} \in \mathcal{S}$, Lemma 6.1 shows that we cannot have $\hat{\tau}=\varrho^{+}$. Thus, $\hat{\tau}$ has to be a face of $\varrho^{-}$. The inclusions

$$
\hat{\tau} \subseteq \varrho^{-} \subseteq \varrho \subseteq \sigma \subseteq \tau
$$

with $\hat{\tau}, \tau \in \mathcal{S}$ and $\sigma \notin \mathcal{S}$ contradict the closedness of Ex $\mathcal{S}$ in Definition 3.3.

Now assume that $\varrho=\sigma^{+} \neq \sigma^{-}$. Since $\varrho \in \mathcal{X}^{\varepsilon}(x), \varrho$ has to be a face of $\sigma$, so we get $\sigma^{+}=\sigma \notin \mathcal{S}$ as well as $\sigma^{-} \notin \mathcal{S}$. Moreover, in this case,

$$
y \in F_{\varrho}(x)=B_{\varrho}=B_{\sigma^{+}} \subseteq \sigma^{+}=\sigma .
$$

Hence, $\sigma_{\text {min }}^{0}(y) \subseteq \sigma$. Given that $y \in \operatorname{cl}\langle\hat{\tau}\rangle_{\zeta}$, we obtain

$$
\hat{\tau} \subseteq \sigma_{\max }^{\zeta}(y) \subseteq \sigma_{\min }^{0}(y) \subseteq \sigma \subseteq \tau
$$

This contradicts Corollary 3.5 because $\hat{\tau}, \tau \in \mathcal{S}$ and $\sigma \notin \mathcal{S}$.

Next assume that $\varrho=\sigma^{-} \neq \sigma^{+}$. Then, $y \in F_{\varrho}(x)=C_{\varrho} \subseteq A_{\varrho}$. Hence, the inclusion $y \in A_{\varrho} \cap \operatorname{cl}\langle\hat{\tau}\rangle_{\zeta}$ holds, and we get a contradiction as in the first case. 
The last possible case is $\varrho=\sigma^{-}=\sigma \in$ Fix $\mathcal{V}$. Then, $y \in \varrho$. Since,

$$
\hat{\tau} \subseteq \sigma_{\min }^{0}(y) \subseteq \sigma
$$

we get the inclusions

$$
\hat{\tau} \subseteq \varrho \subseteq \sigma \subseteq \tau
$$

and a contradiction is reached as before.

Lemma 6.3 (see [19, Lemma 4.10]) The image $F(x)$ can be expressed alternatively as

$$
F(x)=F_{\sigma_{\max }^{\varepsilon}(x)} \cup \bigcup_{\tau \in \mathcal{T}^{\varepsilon}(x)} F_{\tau}(x),
$$

where

$$
\mathcal{T}^{\varepsilon}(x):=\left\{\tau \in \mathcal{X}^{\varepsilon}(x) \backslash\left\{\sigma_{\max }^{\varepsilon}(x)\right\} \mid \tau=\tau^{-} \text {and } \tau^{+} \notin \mathrm{Cl} \sigma_{\max }^{\varepsilon}(x)\right\} .
$$

Furthermore, every $\tau \in \mathcal{T}^{\varepsilon}(x)$ automatically satisfies $\tau \in \operatorname{dom} \mathcal{V} \backslash$ Fix $\mathcal{V}$.

Lemma 6.4 We have

$$
F\left(N_{\delta}\right) \cap N_{\delta} \subseteq\langle\mathcal{S}\rangle
$$

Consequently, Inv $N_{\delta} \subseteq\langle\mathcal{S}\rangle$.

Proof We begin by showing that

$$
F\left(N_{\delta}\right) \cap N_{\delta} \subseteq|\mathcal{S}|
$$

Let $y \in F\left(N_{\delta}\right) \cap N_{\delta}$ with $y \in F(x)$ for some $x \in N_{\delta}$. Then, there exists a $\tau \in \mathcal{S}$ and a $\hat{\tau} \in \mathcal{S}$ such that $x \in \operatorname{cl}\langle\tau\rangle_{\delta}$ and $y \in \operatorname{cl}\langle\hat{\tau}\rangle_{\delta}$. Let $\sigma_{0}:=\sigma_{\text {min }}^{0}(x)$ and $\sigma:=\sigma_{\text {max }}^{\varepsilon}(x)$. By Lemma 4.1 and by Corollary 4.4, we have

$$
\begin{aligned}
\tau \subseteq \sigma_{\max }^{\delta}(x) \subseteq \sigma_{\text {min }}^{0}(x) & =\sigma_{0}, \\
\sigma & =\sigma_{\text {max }}^{\varepsilon}(x) \subseteq \sigma_{\text {min }}^{0}(x)=\sigma_{0},
\end{aligned}
$$

and

$$
\sigma_{\max }^{\varepsilon}(x) \subseteq \sigma_{\min }^{\delta}(x) \subseteq \tau
$$

This implies that $\sigma \subseteq \tau \subseteq \sigma_{0}$. We have $F(x) \cap N_{\delta} \neq \varnothing$ because $y \in F(x) \cap N_{\delta}$. From Lemma 6.2, we get $\sigma \in \mathcal{S}$. Hence, $\sigma^{ \pm} \in \mathcal{S}$. Since $y \in F(x)$, we now consider the following cases resulting from Lemma 6.3.

Assume first that $y \in F_{\varrho}(x)$ for a simplex $\varrho \in \mathcal{T}^{\varepsilon}(x)$. Then, $\varrho \neq \sigma, \varrho=\varrho^{-}$, and $\varrho^{+} \notin \mathrm{Cl} \sigma$. We will show that $\varrho \in \mathcal{S}$. To see this assume the contrary. Then,

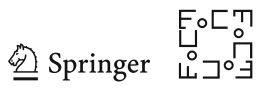


one obtains $\varrho \neq \sigma^{-}, \varrho \neq \sigma^{+}$, so $F_{\varrho}(x)=A_{\varrho}$ and $y \in A_{\varrho} \cap \operatorname{cl}\langle\hat{\tau}\rangle_{\delta}$ with $\hat{\tau} \in \mathcal{S}$. Lemma 6.1 implies that $\hat{\tau}=\varrho^{+}$or $\hat{\tau}$ is a face of $\varrho$. If $\hat{\tau}=\varrho^{+}$, then $\varrho^{+} \in \mathcal{S}$, which implies that $\varrho \in \mathcal{S}$, a contradiction. If $\hat{\tau}$ is a face of $\varrho$, we get the inclusions

$$
\hat{\tau} \subseteq \varrho \subseteq \sigma_{\max }^{\varepsilon}(x)=\sigma \in \mathcal{S}
$$

with $\hat{\tau}, \sigma \in \mathcal{S}$ and $\varrho \notin \mathcal{S}$. As in the proof of Lemma 6.2, this contradicts that $\mathcal{S}$ is an isolated invariant set. Thus, $\varrho \in \mathcal{S}$. Then, $\varrho^{+} \in \mathcal{S}$ and $y \in F_{\varrho}(x) \subseteq \varrho^{+} \subseteq|\mathcal{S}|$.

Now assume that $y \in F_{\sigma}(x)=F_{\sigma_{\max }^{\varepsilon}(x)}(x)$. Then, $F_{\sigma}(x)$ can either be $B_{\sigma}$, or $C_{\sigma}$, or $\sigma$. All these sets are contained in $\sigma^{+} \in \mathcal{S}$; hence, also in this case $y \in|\mathcal{S}|$ and (23) is proved. By Proposition 3.1(ii), in order to conclude the proof, it suffices to show that

$$
F\left(N_{\delta}\right) \cap N_{\delta} \cap|\operatorname{Ex} \mathcal{S}|=\varnothing .
$$

Assume the contrary. Then, there exists a point $z \in F\left(N_{\delta}\right) \cap N_{\delta}$ with $z \in \stackrel{\circ}{\tilde{\sigma}}$ for some $\tilde{\sigma} \in \operatorname{Ex} \mathcal{S}$. Since $z \in N_{\delta}$, there exists a $\tilde{\tau} \in \mathcal{S}$ such that $z \in \operatorname{cl}\langle\tilde{\tau}\rangle_{\delta}$. We get the inclusions

$$
\tilde{\tau} \subseteq \sigma_{\max }^{\delta}(z) \subseteq \sigma_{\min }^{0}(z)=\tilde{\sigma}
$$

with $\tilde{\tau} \in \mathcal{S}$. Since $\tilde{\sigma} \in \operatorname{Ex} \mathcal{S}$, this contradicts the closedness of Ex $\mathcal{S}$ and completes the proof.

Lemma 6.5 Assume $\mathcal{S}$ is an isolated invariant set for $\Pi_{\mathcal{V}}$ in the sense of Definition 3.3, and consider the set $N=N_{\delta} \subseteq X=|\mathcal{X}|$ given by (18). Then, $x \in \operatorname{bd} N_{\delta}$ if and only if

$$
\mathcal{X}^{\delta}(x) \cap \mathcal{S} \neq \varnothing \quad \text { and } \quad \mathcal{X}^{\delta}(x) \backslash \mathcal{S} \neq \varnothing .
$$

Proof The fact that $x \in$ bd $N_{\delta}$ implies (24) is shown in [19, Lemma 5.5]. The reverse implication is an easy consequence of Proposition 4.3.

Lemma 6.6 For any $x \in N_{\delta^{\prime}} \cap$ bd $N_{\delta}$, we have $\sigma_{\text {max }}^{\varepsilon}(x) \notin \mathcal{S}$.

Proof Since $x \in$ bd $N_{\delta}$, Lemma 6.5 implies that there exists a $\sigma_{1} \in \mathcal{X}^{\delta}(x) \backslash \mathcal{S}$. Moreover, let $\sigma_{0}=\sigma_{\text {min }}^{0}(x)$ and $\sigma=\sigma_{\text {max }}^{\varepsilon}(x)$. By Corollary 4.4 we then obtain the inclusions $\sigma=\sigma_{\text {max }}^{\varepsilon}(x) \subseteq \sigma_{\text {min }}^{\delta}(x) \subseteq \sigma_{1} \subseteq \sigma_{\max }^{\delta}(x)$. In addition, we further have $x \in N_{\delta^{\prime}}$. Hence, there exists a simplex $\tau^{\prime} \in \mathcal{S}$ with $x \in \operatorname{cl}\left\langle\tau^{\prime}\right\rangle_{\delta^{\prime}}$. This implies that $\sigma_{\text {max }}^{\delta}(x) \subseteq \sigma_{\text {min }}^{\delta^{\prime}}(x) \subseteq \tau^{\prime} \subseteq \sigma_{\text {min }}^{\delta^{\prime}}(x)$. Together, this gives $\sigma \subseteq \sigma_{1} \subseteq \tau^{\prime}$, where both $\sigma_{1} \notin \mathcal{S}$ and $\tau^{\prime} \in \mathcal{S}$ hold. Therefore, Corollary 3.5 implies that $\sigma \notin \mathcal{S}$, which completes the proof.

\subsection{Property (a)}

Lemma 6.7 The sets $P_{1}, P_{2}$ given by (20) and $N=N_{\delta}$ given by (18) satisfy property (a) in Definition 3.6. 
Proof Let $i=1$. We know from Lemma 6.4 that

$$
F\left(P_{1}\right) \cap N_{\delta} \subseteq F\left(N_{\delta}\right) \cap N_{\delta} \subseteq\langle\mathcal{S}\rangle
$$

and obviously $F\left(P_{1}\right) \cap N_{\delta} \subseteq N_{\delta}$. We argue by contradiction. Suppose that there exists an $x \in F\left(P_{1}\right) \cap N_{\delta}$ with $x \notin N_{\delta^{\prime}}$.

Since $x \in N_{\delta}$, we have $x \in \operatorname{cl}\langle\tau\rangle_{\delta}$ for some $\tau \in \mathcal{S}$. Moreover, since $x \notin N_{\delta^{\prime}}$, we have $x \in \operatorname{cl}\left\langle\tau^{\prime}\right\rangle_{\delta^{\prime}}$ for some $\tau^{\prime} \notin \mathcal{S}$. Now let $\sigma_{0}=\sigma_{\text {min }}^{0}(x)$. Then, one obtains the inclusions $\tau \subseteq \sigma_{\text {max }}^{\delta}(x) \subseteq \sigma_{\text {min }}^{\delta^{\prime}}(x) \subseteq \tau^{\prime} \subseteq \sigma_{\text {max }}^{\delta^{\prime}}(x) \subseteq \sigma_{0}$, where $\tau \in \mathcal{S}$ and $\tau^{\prime} \notin \mathcal{S}$. Therefore, we get from Corollary 3.5 that $\sigma_{0} \notin \mathcal{S}$. This shows that $x \notin\langle\mathcal{S}\rangle$ because by (7) we also have $x \in \stackrel{\circ}{\sigma}_{0}$. Since $x \in F\left(P_{1}\right) \cap N_{\delta}$, this contradicts (25) and proves the claim for $i=1$.

Consider now the case $i=2$, and let $x \in P_{2}=N_{\delta^{\prime}} \cap$ bd $N_{\delta}$. Then, Lemma 6.6 implies $\sigma_{\max }^{\varepsilon}(x) \notin \mathcal{S}$. Since $x \in N_{\delta}$, Lemma 6.2 shows that $F(x) \cap N_{\delta}=\varnothing \subseteq P_{2}$, and the conclusion follows.

\subsection{Property (d)}

Lemma 6.8 We have

$$
P_{1} \backslash P_{2}=N_{\delta^{\prime}} \cap \operatorname{int} N_{\delta}
$$

As a consequence, property (d) in Definition 3.6 is satisfied.

Proof Let $x \in P_{1} \backslash P_{2}$ be arbitrary. Since $x \in P_{1}$, we have $x \in N_{\delta}$ and $x \in N_{\delta^{\prime}}$. Since $x \notin P_{2}$, either $x \notin$ bd $N_{\delta}$ or $x \notin N_{\delta^{\prime}}$. The second case is excluded; hence, we have $x \notin$ bd $N_{\delta}$. It follows that $x \in$ int $N_{\delta}$, and therefore also that $x \in N_{\delta^{\prime}} \cap$ int $N_{\delta}$.

Conversely, let $x \in N_{\delta^{\prime}} \cap$ int $N_{\delta} \subseteq P_{1}$. Then, both $x \notin$ bd $N_{\delta}$ and $x \notin P_{2}$ are satisfied. It follows that $x \in P_{1} \backslash P_{2}$.

Now, property (d) trivially follows from the inclusion $N_{\delta^{\prime}} \cap$ int $N_{\delta} \subseteq$ int $N_{\delta}$.

\subsection{Property (c)}

Lemma 6.9 We have

$$
\operatorname{Inv} N_{\delta} \subseteq \operatorname{int}\left(P_{1} \backslash P_{2}\right)
$$

In other words, property (c) in Definition 3.6 is satisfied.

Proof According to Theorem 5.1, we have

$$
\operatorname{Inv} N_{\delta} \subseteq \operatorname{int} N_{\delta}
$$

We will show in the following that also

$$
\operatorname{Inv} N_{\delta} \subseteq \operatorname{int} N_{\delta^{\prime}} .
$$

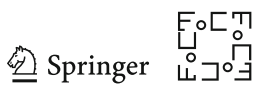


We argue by contradiction. Suppose that Inv $N_{\delta} \backslash$ int $N_{\delta^{\prime}} \neq \varnothing$ and let $x \in \operatorname{Inv} N_{\delta}$ be such that $x \notin$ int $N_{\delta^{\prime}}$. If $x \in$ bd $N_{\delta^{\prime}}$, then Lemma 6.5 shows that there exists a simplex $\tau^{\prime} \notin \mathcal{S}$ with $x \in \operatorname{cl}\left\langle\tau^{\prime}\right\rangle_{\delta^{\prime}}$. It is clear that if $x \notin N_{\delta^{\prime}}$, such a $\tau^{\prime}$ also exists. According to Lemma 6.4, we have $x \in\langle\mathcal{S}\rangle$, that is, there exists a simplex $\sigma \in \mathcal{S}$ with $x \in \stackrel{\circ}{\sigma}$. By (7) we have $\sigma_{\min }^{0}(x)=\sigma$. As before, we obtain inclusions

$$
\sigma_{\max }^{\varepsilon}(x) \subseteq \sigma_{\min }^{\delta^{\prime}}(x) \subseteq \tau^{\prime} \subseteq \sigma_{\max }^{\delta^{\prime}}(x) \subseteq \sigma_{\min }^{0}(x)=\sigma
$$

with $\tau^{\prime} \notin \mathcal{S}$ and $\sigma \in \mathcal{S}$, and Corollary 3.5 implies that $\sigma_{\text {max }}^{\varepsilon}(x) \notin \mathcal{S}$. Due to our assumption, we have $x \in \operatorname{Inv} N_{\delta} \subseteq N_{\delta}$, and Lemma 6.2 gives $F(x) \cap N_{\delta}=\varnothing$. Hence, $x \notin \operatorname{Inv} N_{\delta}$, which is a contradiction, and thus proves (27).

The inclusions (26) and (27) give

$$
\operatorname{Inv} N_{\delta} \subseteq \operatorname{int} N_{\delta^{\prime}} \cap \operatorname{int} N_{\delta}=\operatorname{int}\left(N_{\delta^{\prime}} \cap \operatorname{int} N_{\delta}\right)=\operatorname{int}\left(P_{1} \backslash P_{2}\right),
$$

which completes the proof.

For the next result, we need the following two simple observations. If $A$ and $B$ are closed subsets of $X$, then

$$
\operatorname{bd}(A \cap B) \subseteq(\mathrm{bd} A \cap B) \cup(A \cap \operatorname{bd} B)
$$

If $A$ is a closed subset of $X$, then

$$
\operatorname{bd}_{F}(A) \subseteq A \cap F(A) .
$$

The first observation is straightforward. In order to verify the second one, it is clear that $\operatorname{bd}_{F}(A) \subseteq \operatorname{cl} A=A$. Since $F(A) \backslash A \subseteq F(A)$, we get

$$
\operatorname{cl}(F(A) \backslash A) \subseteq \operatorname{cl} F(A)=F(A),
$$

because the map $F$ is upper semicontinuous and the set $X$ is compact. These two inclusions immediately give (29).

\subsection{Property (b)}

Lemma 6.10 For $P_{1}$ and $P_{2}$ defined by (20), we have

$$
\operatorname{bd}_{F}\left(P_{1}\right) \subseteq P_{2}
$$

In other words, property (b) in Definition 3.6 is satisfied.

Proof One can easily see that $\operatorname{bd}_{F}\left(P_{1}\right) \subseteq \operatorname{bd}\left(P_{1}\right)$. Together with (28), this further implies

$$
\operatorname{bd}_{F}\left(P_{1}\right) \subseteq \operatorname{bd}\left(P_{1}\right)=\operatorname{bd}\left(N_{\delta} \cap N_{\delta^{\prime}}\right)
$$




$$
\begin{aligned}
& \subseteq\left(N_{\delta^{\prime}} \cap \mathrm{bd} N_{\delta}\right) \cup\left(N_{\delta} \cap \mathrm{bd} N_{\delta^{\prime}}\right) \\
& =P_{2} \cup\left(N_{\delta} \cap \mathrm{bd} N_{\delta^{\prime}}\right) .
\end{aligned}
$$

Thus, if we can show that

$$
\operatorname{bd}_{F}\left(P_{1}\right) \cap\left(N_{\delta} \cap \operatorname{bd} N_{\delta^{\prime}}\right)=\varnothing,
$$

then the proof is complete. We prove this by contradiction. Assume that there exists an $x \in \operatorname{bd}_{F}\left(P_{1}\right) \cap\left(N_{\delta} \cap\right.$ bd $\left.N_{\delta^{\prime}}\right)$. Since $x \in \operatorname{bd}_{F}\left(P_{1}\right)$, by (29) we get

$$
x \in P_{1} \cap F\left(P_{1}\right) \subseteq N_{\delta} \cap F\left(N_{\delta}\right) .
$$

Thus, due to Lemma 6.4, we have the inclusion $x \in\langle\mathcal{S}\rangle$. It follows that there exists a simplex $\sigma \in \mathcal{S}$ with $x \in \stackrel{\circ}{\sigma}$ and by (7) $\sigma=\sigma_{\text {min }}^{0}(x)$.

Since $x \in N_{\delta}$, we get a simplex $\tau \in \mathcal{S}$ such that $x \in \operatorname{cl}\langle\tau\rangle_{\delta}$ and since $x \in \operatorname{bd} N_{\delta^{\prime}}$, by Lemma 6.5 we also get a simplex $\tau^{\prime} \notin \mathcal{S}$ such that $x \in \operatorname{cl}\left\langle\tau^{\prime}\right\rangle_{\delta^{\prime}}$. Now, Lemma 4.1 and Corollary 4.4 imply

$$
\tau \subseteq \sigma_{\max }^{\delta}(x) \subseteq \sigma_{\min }^{\delta^{\prime}}(x) \subseteq \tau^{\prime} \subseteq \sigma_{\max }^{\delta^{\prime}}(x) \subseteq \sigma_{\min }^{0}(x)=\sigma
$$

Since $\tau, \sigma \in \mathcal{S}$ and $\tau^{\prime} \notin \mathcal{S}$, this contradicts, in combination with Corollary 3.5, the fact that $\mathcal{S}$ is an isolated invariant set-and the proof is complete.

Theorem 5.2 is now an immediate consequence of Lemmas 6.7, 6.8, 6.9, 6.10.

\section{Proof of Theorem 5.3}

In this section we prove Theorem 5.3. Since the Leray reduction of an identity is clearly the same identity, it suffices to prove that the index map $I_{P}$ is the identity map. We achieve this by constructing an acyclic-valued and upper semicontinuous map $G$ whose graph contains both the graph of $F$ and the graph of the identity. The map $G$ is constructed by gluing two multivalued and acyclic maps. One of these, the map $\tilde{F}$ defined below, is a modification of our map $F$, while the second map $D$ contains the identity.

\subsection{The $\operatorname{Map} \tilde{\boldsymbol{F}}$}

For $x \in X$ and $\sigma \in \mathcal{X}^{\varepsilon}(x)$, we define

$$
\tilde{F}_{\sigma}(x):=F_{\sigma}(x) \cup A_{\sigma} .
$$

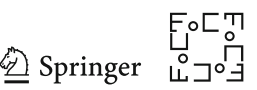


One can then easily verify that

$$
\tilde{F}_{\sigma}(x)= \begin{cases}A_{\sigma} & \text { if } F_{\sigma}(x)=C_{\sigma} \\ \sigma^{+} & \text {if } F_{\sigma}(x)=B_{\sigma} \\ F_{\sigma}(x) & \text { otherwise, }\end{cases}
$$

and that the inclusion

$$
\sigma \subseteq \tilde{F}_{\sigma}(x)
$$

holds. We will show that the auxiliary map $\tilde{F}: X \multimap X$ given by

$$
\tilde{F}(x):=\bigcup_{\sigma \in \mathcal{X}^{\varepsilon}(x)} \tilde{F}_{\sigma}(x) .
$$

is acyclic-valued. For this we need a few auxiliary results.

Lemma 7.1 For any $x \in X$ and $\sigma:=\sigma_{\max }^{\varepsilon}(x)$, we have

$$
\tilde{F}(x)=A_{\sigma} \cup F(x) .
$$

Proof It is straightforward to observe that the right-hand side of (34) is contained in the left-hand side. To prove the opposite inclusion, take a $y \in \tilde{F}(x)$ and select a simplex $\tau \in \mathcal{X}^{\varepsilon}(x)$ such that $y \in \tilde{F}_{\tau}(x)$. In particular, $\tau \subseteq \sigma$. Note that if $\tau=\sigma$, then

$$
\tilde{F}_{\tau}(x)=\tilde{F}_{\sigma}(x)=F_{\sigma}(x) \cup A_{\sigma} \subseteq F(x) \cup A_{\sigma} .
$$

According to (15), the value $F_{\tau}(x)$ may be $A_{\tau}, B_{\tau}, C_{\tau}$ or $\tau$. Assume first that we have $F_{\tau}(x)=A_{\tau}$. Then,

$$
\tilde{F}_{\tau}(x)=F_{\tau}(x) \cup A_{\tau}=F_{\tau}(x) \subseteq F(x) \subseteq F(x) \cup A_{\sigma} .
$$

Next, consider the case $F_{\tau}(x)=B_{\tau}$. Then, $\tau=\sigma^{+} \neq \sigma^{-}$. Since $\tau \subseteq \sigma$, we cannot have $\sigma=\sigma^{-}$. Hence, $\sigma=\sigma^{+}=\tau$ and estimation (35) applies. Assume in turn that $F_{\tau}(x)=C_{\tau}$. Then, $\tau=\sigma^{-} \neq \sigma^{+}, A_{\tau}=A_{\sigma}$ and

$$
\tilde{F}_{\tau}(x)=C_{\tau} \cup A_{\tau}=A_{\tau}=A_{\sigma} \subseteq F(x) \cup A_{\sigma} .
$$

Finally, if $F_{\tau}(x)=\tau$, then $\tau=\sigma^{-}=\sigma^{+}=\sigma$ and again (35) applies.

Proposition 7.2 Let $x \in X$ and let $\sigma:=\sigma_{\max }^{\varepsilon}(x)$. For any simplex $\tau \in \mathcal{T}^{\varepsilon}(x)$, where $\mathcal{T}^{\varepsilon}(x)$ is given as in (22), we have

$$
A_{\tau} \cap A_{\sigma}=\tau \cap \sigma^{-} .
$$

Proof We have $\tau \cap \sigma^{-} \subseteq \sigma^{-} \subseteq A_{\sigma}$ and $\tau \cap \sigma^{-} \subseteq \tau=\tau^{-} \subseteq A_{\tau}$, which shows that the right-hand side of (36) is contained in the left-hand side. Observe that for any simplex $\varrho \neq \sigma^{+}$, we have $\varrho \cap A_{\sigma} \subseteq \sigma^{-}$. We cannot have $\tau^{+}=\sigma^{+}$, because then 
either $\sigma=\sigma^{-}=\tau^{-}=\tau$ or $\tau^{+}=\sigma^{+}=\sigma \in \mathrm{Cl} \sigma$, in both cases contradicting the inclusion $\tau \in \mathcal{T}^{\varepsilon}(x)$. Thus, $A_{\tau} \cap A_{\sigma} \subseteq \tau^{+} \cap A_{\sigma} \subseteq \sigma^{-}$. It follows that we must have $A_{\tau} \cap A_{\sigma} \subseteq \sigma^{-} \cap \tau^{+} \subseteq \sigma \cap \tau^{+}$. The simplex $\sigma \cap \tau^{+}$must be a proper face of $\tau^{+}$ because otherwise $\tau^{+}$is a face of $\sigma$ which contradicts $\tau^{+} \notin \mathrm{Cl} \sigma$. But, $\tau \subseteq \sigma \cap \tau^{+}$ and $\tau=\tau^{-}$is a face of $\tau^{+}$of codimension one. It follows that $\sigma \cap \tau^{+}=\tau$ which proves (36).

The following proposition is implicitly proved in the second to last paragraph of the proof of [19, Theorem 4.12].

Proposition 7.3 For any $\tau \in \mathcal{T}^{\varepsilon}(x)$ we have $F_{\tau}(x)=A_{\tau}$.

Lemma 7.4 For an $x \in X$ and $\sigma:=\sigma_{\max }^{\varepsilon}(x)$, we have

$$
A_{\sigma} \cap F(x)=A_{\sigma} \cap F_{\sigma}(x) .
$$

Proof Obviously, the right-hand side is contained in the left-hand side. To prove the opposite inclusion, choose a $y \in F(x) \cap A_{\sigma}$ and select a simplex $\tau$ such that $y \in F_{\tau}(x)$. It suffices to show that $y \in F_{\sigma}(x)$. By Lemma 6.3 we may assume that either $\tau=\sigma$ or $\tau \in \mathcal{T}^{\varepsilon}(x)$. If $\tau=\sigma$, the inclusion is obvious. Hence, assume that $\tau \in \mathcal{T}^{\varepsilon}(x)$. By Corollary 4.4 we have $\tau \subseteq \sigma$. This means that $\tau \subsetneq \sigma, \tau=\tau^{-}$and $\tau^{+} \notin \mathrm{Cl} \sigma$. From Proposition 7.3 we get $F_{\tau}(x)=A_{\tau}$. Therefore,

$$
y \in A_{\tau} \cap A_{\sigma} \subseteq \tau^{+} \cap A_{\sigma} \subseteq \tau^{+} \cap \sigma^{+} .
$$

If $\sigma=\sigma^{+}=\sigma^{-}$, then $y \in A_{\sigma}=\sigma=F_{\sigma}(x)$, and hence, the inclusion holds. Thus, consider the case $\sigma^{+} \neq \sigma^{-}$. By Proposition 7.2 we get $y \in \tau \cap \sigma^{-}$. We cannot have $\tau \cap \sigma^{-}=\sigma^{-}$, because then $\sigma^{-} \subseteq \tau \subsetneq \sigma, \tau^{-}=\tau=\sigma^{-}, \tau^{+}=\sigma^{+}$and $\tau \neq \sigma$ implies $\tau^{+}=\sigma^{+}=\sigma, \tau^{+} \in \mathrm{Cl} \sigma$, a contradiction. Hence, $\tau \cap \sigma^{-}$is a proper face of the simplex $\sigma^{-}$. Therefore, $\tau \cap \sigma^{-} \subseteq C_{\sigma} \subseteq F_{\sigma}(x)$.

Theorem 7.5 The map $\tilde{F}$ is upper semicontinuous and acyclic-valued.

Proof The upper semicontinuity of the map $\tilde{F}$ is an immediate consequence of formula (33) and Lemma 4.2. To show that $\tilde{F}$ is acyclic-valued fix an $x \in X$. By (34), $\tilde{F}(x)=A_{\sigma} \cup F(x)$. The set $A_{\sigma}$ is acyclic by Lemma 4.5 and the set $F(x)$ is acyclic by Theorem 4.6. Moreover, $A_{\sigma} \cap F(x)=A_{\sigma} \cap F_{\sigma}(x)$ by (37). Hence, due to (15) the intersection $A_{\sigma} \cap F(x)$ is either $A_{\sigma}$ or $C_{\sigma}$, hence also acyclic. Moreover, $F(x)$ and $A_{\sigma}$ are closed subcomplexes of the $\gamma$-cell decomposition of $X$. Therefore, it follows from the Mayer-Vietoris theorem that $\tilde{F}(x)$ is acyclic.

Proposition 7.6 The weak index pair $P$ is positively invariant with respect to $\tilde{F}$ and $N_{\delta}$, that is, we have

$$
\tilde{F}\left(P_{i}\right) \cap N_{\delta} \subseteq P_{i} \quad \text { for } \quad i=1,2
$$

Proof The proof is analogous to the proof of Lemma 6.7.

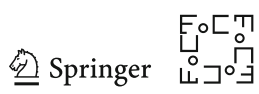




\subsection{The Map $\tilde{D}$}

We define a multivalued map $D: X \multimap X$, by letting

$$
D(x):=\operatorname{conv}\left(\{x\} \cup \sigma_{\max }^{\varepsilon}(x)\right),
$$

where conv $A$ denotes the convex hull of $A$. Note that the above definition is well posed because both $\{x\}$ and $\sigma_{\max }^{\varepsilon}(x)$ are subsets of the same simplex $\sigma_{\min }^{0}(x)$.

In order to show that $D$ is upper semicontinuous, we need the following lemma.

\section{Lemma 7.7 The mapping}

$$
X \ni x \mapsto \sigma_{\max }^{\varepsilon}(x) \subseteq X
$$

is strongly upper semicontinuous, that is, for every $x \in X$ there exists a neighborhood $V$ of $x$ such that for each $y \in V$ we have $\sigma_{\text {max }}^{\varepsilon}(y) \subseteq \sigma_{\max }^{\varepsilon}(x)$.

Proof By Lemma 4.2, we can choose a neighborhood $V$ of $x$ in such a way that $\mathcal{X}^{\varepsilon}(y) \subseteq \mathcal{X}^{\varepsilon}(x)$ for $y \in V$. In particular, $\sigma_{\text {max }}^{\varepsilon}(y) \in \mathcal{X}^{\varepsilon}(y) \subseteq \mathcal{X}^{\varepsilon}(x)$. By Corollary 4.4, we obtain $\sigma_{\text {max }}^{\varepsilon}(y) \subseteq \sigma_{\text {max }}^{\varepsilon}(x)$.

Proposition 7.8 The mapping $D$ is upper semicontinuous and has non-empty and contractible values.

Proof Since the values of $D$ are convex, they are obviously contractible. To see that $D$ is upper semicontinuous, fix $\varepsilon>0$. By Lemma 7.7 we can find a neighborhood $V$ of $x$ such that $\sigma_{\max }^{\varepsilon}(y) \subseteq \sigma_{\max }^{\varepsilon}(x)$. Let $B(x, \varepsilon)$ denote the $\varepsilon$-ball around $x$, let $y \in B(x, \varepsilon) \cap V$, and fix a point $z \in D(y)$. Then, $z=t y+(1-t) \bar{y}$ for a $t \in[0,1]$ and $\bar{y} \in \sigma_{\max }^{\varepsilon}(y)$. Let $z^{\prime}:=t x+(1-t) \bar{y}$. Since $\sigma_{\max }^{\varepsilon}(y) \subseteq \sigma_{\max }^{\varepsilon}(x)$, we have $z^{\prime} \in D(x)$. Moreover, the estimate $\left\|z-z^{\prime}\right\|=t\|y-x\| \leq\|y-x\|<\varepsilon$ holds. It follows that $z \in B(D(x), \varepsilon)$. Hence, $D(y) \subseteq B(D(x), \varepsilon)$, which proves the upper semicontinuity of $D$.

Lemma 7.9 Let $0<\zeta<\varepsilon$. For any $x \in X$ and any $y \in D(x), y \neq x$, we have

$$
\sigma_{\max }^{\zeta}(y) \subseteq \sigma_{\min }^{\zeta}(x)
$$

Proof Let $x \in X$ and $y \in D(x)$, with $y \neq x$, be fixed. Then, $y=\alpha x+(1-\alpha) x_{\sigma}$ for some $x_{\sigma} \in \sigma_{\max }^{\varepsilon}(x)$ and $\alpha \in[0,1)$. Consider a vertex $v \notin \sigma_{\text {min }}^{\zeta}(x)$. By Lemma 4.1 we have $\sigma_{\max }^{\varepsilon}(x) \subseteq \sigma_{\min }^{\zeta}(x)$, which shows that $v \notin \sigma_{\text {max }}^{\varepsilon}(x)$. Therefore,

$$
\begin{aligned}
t_{v}(y) & =\alpha t_{v}(x)+(1-\alpha) t_{v}\left(x_{\sigma}\right) \\
& =\alpha t_{v}(x)<t_{v}(x) \leq \zeta,
\end{aligned}
$$

which implies $v \notin \sigma_{\max }^{\zeta}(y)$, and the inclusion $\sigma_{\max }^{\zeta}(y) \subseteq \sigma_{\min }^{\zeta}(x)$ follows. 
Proposition 7.10 The weak index pair $P$ is positively invariant with respect to $D$ and $N_{\delta}$, that is, we have

$$
D\left(P_{i}\right) \cap N_{\delta} \subseteq P_{i} \quad \text { for } \quad i=1,2 .
$$

Proof We begin with the proof for the case $i=1$. Fix an $x \in P_{1}=N_{\delta} \cap N_{\delta^{\prime}}$. Then, $x \in \operatorname{cl}\langle\tau\rangle_{\delta}$ and $x \in \operatorname{cl}\left\langle\tau^{\prime}\right\rangle_{\delta^{\prime}}$ for some $\tau, \tau^{\prime} \in \mathcal{S}$. Consider a $y \in D(x) \cap N_{\delta}$. For $y=x$ inclusion (39) trivially holds. Therefore, assume $y \neq x$. Since $y \in N_{\delta}$, there exists a simplex $\eta \in \mathcal{S}$ with $y \in \operatorname{cl}\langle\eta\rangle_{\delta}$. Consider a simplex $\eta^{\prime} \in \mathcal{X}$ such that $y \in \operatorname{cl}\left\langle\eta^{\prime}\right\rangle_{\delta^{\prime}}$. By Lemma 7.9 we obtain $\eta^{\prime} \subseteq \sigma_{\max }^{\delta^{\prime}}(y) \subseteq \sigma_{\min }^{\delta^{\prime}}(x) \subseteq \tau^{\prime}$ and Lemma 4.1 together with Corollary 4.4 implies $\eta \subseteq \sigma_{\max }^{\delta}(y) \subseteq \sigma_{\min }^{\delta^{\prime}}(y) \subseteq \eta^{\prime}$. Thus, $\eta \subseteq \eta^{\prime} \subseteq \tau^{\prime}$. Since $\eta \in \mathcal{S}$ and $\tau^{\prime} \in \mathcal{S}$, we get from Corollary 3.5 that $\eta^{\prime} \in \mathcal{S}$. Thus, $y \in N_{\delta^{\prime}}$, which completes the proof for $i=1$.

To proceed with the proof for $i=2$, fix an $x \in P_{2}$ and consider a $y \in D(x) \cap N_{\delta}$. Inclusion (39) is trivial when $y=x$. Hence, assume $y \neq x$. Note that $P_{2} \subseteq N_{\delta}^{\prime}$, therefore $x \in \operatorname{cl}\left\langle\tau^{\prime}\right\rangle_{\delta^{\prime}}$ for some $\tau^{\prime} \in \mathcal{S}$. Since $x \in \mathrm{bd} N_{\delta}$, by Lemma 6.5 there exists a simplex $\tau \notin \mathcal{S}$ such that $x \in \operatorname{cl}\langle\tau\rangle_{\delta}$. According to Lemma 4.1 we then have the inclusion $\tau \subseteq \sigma_{\text {max }}^{\delta}(x) \subseteq \sigma_{\text {min }}^{\delta^{\prime}}(x) \subseteq \tau^{\prime}$, and this yields $\tau \in$ Ex $\mathcal{S}$. Consider a simplex $\eta$ such that $y \in \operatorname{cl}\langle\eta\rangle_{\delta}$. By Lemma 7.9 we have $\eta \subseteq \sigma_{\max }^{\delta}(y) \subseteq \sigma_{\min }^{\delta}(x) \subseteq \tau$. Now, the closedness of Ex $\mathcal{S}$ implies $\eta \in \operatorname{Ex} \mathcal{S}$. Hence, $y \in$ bd $N_{\delta}$ by Lemma 6.5. Observe that by case $i=1$ we also have $y \in P_{1} \subseteq N_{\delta}^{\prime}$. Therefore, $y \in P_{2}$.

\subsection{The Map G}

Define the multivalued map $G: X \multimap X$ by

$$
G(x):=D(x) \cup \tilde{F}(x) .
$$

Proposition 7.11 The following conditions hold:

(i) $G$ is upper semicontinuous,

(ii) $P$ is positively invariant with respect to $G$ and $N_{\delta}$,

(iii) $G$ is acyclic-valued.

Proof The map $G$ inherits properties (i) and (ii) directly from its summands $\tilde{F}$ and $D$ (see Theorem 7.5, Propositions 7.6, 7.8 and 7.10).

To prove (iii), fix an $x \in X$ and note that $\tilde{F}(x)$ is acyclic by Theorem 7.5 , and that $D(x)$ is acyclic by Proposition 7.11(iii). Let $\sigma:=\sigma_{\max }^{\varepsilon}(x)$ and $\sigma^{0}:=\sigma_{\min }^{0}(x)$. Obviously either $x \in \sigma$ or $x \notin \sigma$. To begin with, we consider the case $x \in \sigma$. Then, one has $D(x)=\sigma$. We will show that $D(x) \subseteq \tilde{F}(x)$. Indeed, if $\sigma=\sigma^{-}$, then we have $D(x)=\sigma=\sigma^{-} \subseteq A_{\sigma} \subseteq \tilde{F}(x)$ by Lemma 7.1. If $\sigma \neq \sigma^{-}$, then one has the equality $\sigma=\sigma^{+}$. In that case $F_{\sigma}(x)=B_{\sigma}$ and by (31) we get $\tilde{F}_{\sigma}(x)=\sigma^{+}$, which shows that $D(x)=\sigma=\sigma^{+}=\tilde{F}_{\sigma}(x) \subseteq \tilde{F}(x)$. Consequently, if $x \in \sigma$, then we have the equality $G(x)=\tilde{F}(x)$, and this set is acyclic by Theorem 7.5.

Thus, consider the case $x \notin \sigma$. By the Mayer-Vietoris theorem, it suffices to show that $D(x) \cap \tilde{F}(x)$ is acyclic because both sets $D(x)$ and $\tilde{F}(x)$ are closed and acyclic

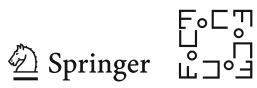


subcomplexes of the $\gamma$-cell decomposition of $X$. To this end we use the following representation

$$
\tilde{F}(x)=F_{\sigma}(x) \cup A_{\sigma} \cup \bigcup_{\tau \in \mathcal{T}^{\varepsilon}(x)} F_{\tau}(x),
$$

which follows immediately from Lemma 7.1 and (21). First, we will show that for every simplex $\tau \in \mathcal{T}^{\varepsilon}(x)$ we have

$$
D(x) \cap F_{\tau}(x) \subseteq D(x) \cap\left(F_{\sigma}(x) \cup A_{\sigma}\right) .
$$

To see this observe that since $x \in \sigma^{0} \backslash \sigma$, it is evident that $\sigma$ is a proper face of the simplex $\sigma^{0}$. Moreover, one can easily observe that

$$
D(x) \subseteq \sigma^{0} \text { and } D(x) \cap\left|\operatorname{Ex} \sigma^{0}\right|=\sigma
$$

Note that from the definition of the collection $\mathcal{T}^{\varepsilon}(x)$ (cf. Lemma 6.3), it follows that any simplex $\tau \in \mathcal{T}^{\varepsilon}(x)$ is a proper face of $\sigma$. Therefore, $\sigma^{0}$ is a coface of $\tau$ of codimension greater than one. Hence, we cannot have $\tau^{+}=\sigma^{0}$. By Proposition 7.3 we have $F_{\tau}(x)=A_{\tau} \subseteq \tau^{+}$. Thus, from $\tau=\tau^{-} \subseteq A_{\tau}$ and (42), we obtain

$$
D(x) \cap F_{\tau}(x) \subseteq \sigma \text { for any } \tau \in \mathcal{T}^{\varepsilon}(x) .
$$

We will now show that

$$
\sigma \subseteq D(x) \cap\left(F_{\sigma}(x) \cup A_{\sigma}\right) .
$$

Obviously, $\sigma \subseteq D(x)$. If $\sigma=\sigma^{-}$, then $\sigma \subseteq A_{\sigma} \subseteq F_{\sigma}(x) \cup A_{\sigma}$. If $\sigma=\sigma^{+}$, then by (15) we have $\sigma \subseteq \sigma^{+} \subseteq F_{\sigma}(x) \cup A_{\sigma}$. Hence, (44) is proved. Formula (41) follows now from (43) and (44). From (41) we immediately obtain that

$$
D(x) \cap \tilde{F}_{\tau}(x)=D(x) \cap\left(F_{\sigma}(x) \cup A_{\sigma}\right) .
$$

Now we distinguish the two complementary cases: $\sigma=\sigma^{+}$and $\sigma=\sigma^{-} \neq \sigma^{+}$. First of all, if $\sigma=\sigma^{+}$, then

$$
D(x) \cap\left(F_{\sigma}(x) \cup A_{\sigma}\right) \subseteq \sigma^{0} \cap \sigma^{+}=\sigma^{0} \cap \sigma=\sigma .
$$

It follows from (44) and (41) that in this case

$$
D(x) \cap \tilde{F}(x)=D(x) \cap\left(F_{\sigma}(x) \cup A_{\sigma}\right)=\sigma
$$

is an acyclic set. We show that the same is true in the second case $\sigma=\sigma^{-} \neq \sigma^{+}$. Now one has $F_{\sigma}(x) \cup A_{\sigma}=C_{\sigma} \cup A_{\sigma}=A_{\sigma}$. Observe that $A_{\sigma}=A^{+} \cup \sigma^{-}$, where

$$
A^{+}:=\left\{y \in \sigma^{+} \mid t_{v}(y) \geq \gamma \text { for } v \in \sigma^{-}\right\}
$$


is a convex set. This, together with (45), shows that

$$
D(x) \cap \tilde{F}(x)=D(x) \cap A_{\sigma}=D(x) \cap\left(A^{+} \cup \sigma^{-}\right)=\left(D(x) \cap A^{+}\right) \cup \sigma^{-} .
$$

The acyclicity of the right-hand side of (46) follows from the Mayer-Vietoris theorem because $D(x) \cap A^{+}, \sigma^{-}$, and $D(x) \cap A^{+} \cap \sigma^{-}=A^{+} \cap \sigma^{-}$are all closed, acyclic subcomplexes of the $\gamma$-cell decomposition of $X$. Therefore, by (46) also in this case the set $D(x) \cap \tilde{F}(x)$ is acyclic. This completes the proof.

We are now able to prove Theorem 5.3.

Proof of Theorem 5.3: By Lemma 7.6 and Proposition 7.10, we can consider the map $G$, given by (40), as a map of pairs

$$
G:\left(P_{1}, P_{2}\right) \multimap\left(T_{1}(P), T_{2}(P)\right) .
$$

Directly from the definition of $G$, it follows that both the inclusion $i: P \rightarrow T(P)$ and $F: P \multimap T(P)$ are selectors of $G$, that is, for any $x \in P_{1}$ we have

$$
x \in G(x) \quad \text { and } \quad F(x) \subseteq G(x) .
$$

Moreover, all of the above maps are acyclic-valued (cf. again Proposition 7.11 and Theorem 4.6). Therefore, it follows from [16, Proposition 32.13(i)] that the identities $H^{*}(F)=H^{*}(G)=H^{*}(i)$ are satisfied. As a consequence, we obtain the desired equality $I_{P}=\mathrm{id}_{H^{*}(P)}$, which completes the proof.

\section{Proof of Theorem 5.4}

In order to prove Theorem 5.4, we first construct an auxiliary pair $\left(Q_{1}, Q_{2}\right)$ and show that $H^{*}\left(P_{1}, P_{2}\right) \cong H^{*}\left(Q_{1}, Q_{2}\right)$. As a second step, we then construct a continuous surjection $\psi:\left(Q_{1}, Q_{2}\right) \rightarrow(|\mathrm{Cl} \mathcal{S}|, \mid$ Ex $\mathcal{S} \mid)$ with contractible preimages and apply the Vietoris-Begle theorem (cf. [39, Chapter 6.9]) to complete the proof.

\subsection{The Pair $\left(Q_{1}, Q_{2}\right)$}

Consider the pair $\left(Q_{1}, Q_{2}\right)$ consisting of the two sets

$$
\begin{aligned}
& Q_{1}:=N_{\delta}(\mathrm{Cl} \mathcal{S}) \cap N_{\delta^{\prime}}(\mathrm{Cl} \mathcal{S}), \\
& Q_{2}:=N_{\delta}(\operatorname{Ex} \mathcal{S}) \cap N_{\delta^{\prime}}(\mathrm{Cl} \mathcal{S}),
\end{aligned}
$$

where $N_{\delta}(\mathcal{A})$ is given by (17). Figure 12 shows an example of such a pair for the isolated invariant set $\mathcal{S}$ presented in Fig. 9.

Proposition 8.1 We have

$$
H^{*}\left(Q_{1}, Q_{2}\right) \cong H^{*}\left(P_{1}, P_{2}\right)
$$

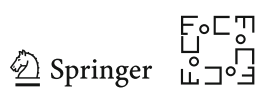




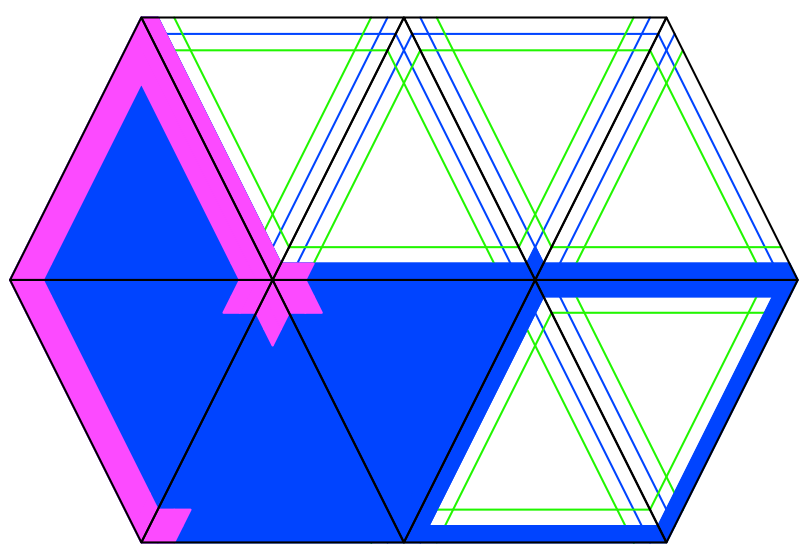

Fig. 12 The pair $Q=\left(Q_{1}, Q_{2}\right)$ associated with the isolating block $N_{\delta}$ from Fig. 9 and the weak index pair $P=\left(P_{1}, P_{2}\right)$ from Fig. 10 . The set $Q_{1}$ is the union of the dark blue and magenta regions, while the subset $Q_{2} \subseteq Q_{1}$ is only the magenta part (Color figure online)

Proof We begin by verifying the two inclusions

$$
P_{i} \subseteq Q_{i} \quad \text { for } \quad i=1,2 .
$$

It is clear that $P_{1} \subseteq Q_{1}$; therefore, we shall verify (48) for $i=2$.

Let $x \in P_{2}$. Then $x \in N_{\delta}$ and by Lemma 6.5, there exists a simplex $\sigma \notin \mathcal{S}$ such that $x \in \operatorname{cl}\langle\sigma\rangle_{\delta}$. On the other hand, $x \in P_{1}$ implies $x \in N_{\delta^{\prime}}(\mathcal{S})$, so we can take $\tau \in \mathcal{S}$ with $x \in \operatorname{cl}\langle\tau\rangle_{\delta^{\prime}}$. For any vertex $v \in \sigma$, we have $t_{v}(x) \geq \delta>\delta^{\prime}$, which shows that $\sigma \subseteq \tau \in \mathcal{S}$. Consequently, $\sigma \in \mathrm{Cl} \mathcal{S}$, which along with $\sigma \notin \mathcal{S}$ implies the inclusions $\sigma \in \operatorname{Ex} \mathcal{S}$ and $x \in N_{\delta}(\operatorname{Ex} \mathcal{S})$. Observe now that we also have $x \in N_{\delta^{\prime}}(\mathrm{Cl} \mathcal{S})$, according to $P_{2} \subseteq N_{\delta^{\prime}}(\mathcal{S}) \subseteq N_{\delta^{\prime}}(\mathrm{Cl} \mathcal{S})$. Thus, $x \in Q_{2}$. The proof of (48) is now complete.

Note that $P_{1}, P_{2}, Q_{1}, Q_{2}$ are compact and $Q_{2} \subseteq Q_{1}$ and $P_{2} \subseteq P_{1}$. Therefore, by the strong excision property of Alexander-Spanier (co)homology (cf. [39, Chapter 6.6]), in order to prove (48), it suffices to verify that $Q_{1} \backslash Q_{2}=P_{1} \backslash P_{2}$.

For this, consider an $x \in Q_{1} \backslash Q_{2}$. Then, $x \in N_{\delta}(\mathrm{Cl} \mathcal{S})$ and $x \notin N_{\delta}(\mathrm{Ex} \mathcal{S})$. Hence, there exists a $\sigma \in \mathrm{Cl} \mathcal{S} \backslash \operatorname{Ex} \mathcal{S}=\mathcal{S}$ with $x \in \operatorname{cl}\langle\sigma\rangle_{\delta}$. It follows that

$$
x \in N_{\delta}(\mathcal{S})
$$

We also have $x \in N_{\delta^{\prime}}(\mathrm{Cl} \mathcal{S})$. In order to show that $x \in P_{1}$, we need to verify the inclusion $x \in N_{\delta^{\prime}}(\mathcal{S})$. Suppose to the contrary that there is a $\tau \in \mathrm{Cl} \mathcal{S} \backslash \mathcal{S}=\operatorname{Ex} \mathcal{S}$ with $x \in \operatorname{cl}\langle\tau\rangle_{\delta^{\prime}}$. Then, for each vertex $v$ of $\sigma$ we have $t_{v}(x) \geq \delta>\delta^{\prime}$, which means that each vertex of $\sigma$ is a vertex of $\tau$. In other words $\sigma \subseteq \tau$. However, $\tau \in \operatorname{Ex} \mathcal{S}$ which, according to the closedness of Ex $\mathcal{S}$, implies $\sigma \in \operatorname{Ex} \mathcal{S}$, a contradiction. Therefore, $x \in N_{\delta^{\prime}}(\mathcal{S})$, and together with (49) this implies the inclusion $x \in P_{1}$. Since $x \notin Q_{2}$, by (48), we further have $x \notin P_{2}$. Consequently, both $x \in P_{1} \backslash P_{2}$ and $Q_{1} \backslash Q_{2} \subseteq P_{1} \backslash P_{2}$ are satisfied.

In order to prove the reverse inclusion, let $x \in P_{1} \backslash P_{2}$ be arbitrary. It is clear that then $x \in Q_{1}$. We need to show that $x \notin Q_{2}$. Suppose the contrary. Then, there 
exists a simplex $\sigma \in \operatorname{Ex} \mathcal{S}$ such that $x \in \operatorname{cl}\langle\sigma\rangle_{\delta}$. It follows that $\sigma \in \mathcal{X}^{\delta}(x) \backslash \mathcal{S}$ and $\mathcal{X}^{\delta}(x) \backslash \mathcal{S} \neq \varnothing$. Since $x \in P_{1} \subseteq N_{\delta}(\mathcal{S})$, we also have $\mathcal{X}^{\delta}(x) \cap \mathcal{S} \neq \varnothing$. Therefore, by Lemma 6.5, we get $x \in$ bd $N_{\delta}(\mathcal{S})$. Yet, we also have $x \in N_{\delta^{\prime}}(\mathcal{S})$ in view of $x \in P_{1}$. Consequently, $x \in$ bd $N_{\delta}(\mathcal{S}) \cap N_{\delta^{\prime}}(\mathcal{S})=P_{2} \subseteq Q_{2}$, which is a contradiction.

\subsection{Auxiliary Maps $\varphi_{\sigma}^{\lambda}$}

Proposition 8.2 For any $\sigma, \tau \in \mathcal{X}$, we have

$$
\begin{aligned}
& \operatorname{cl}\langle\sigma\rangle_{\lambda} \cap \operatorname{cl}\langle\tau\rangle_{\lambda}=\left\{x \in X \mid t_{v}(x)=\lambda \text { for } v \in(\tau \backslash \sigma) \cup(\sigma \backslash \tau),\right. \\
& t_{v}(x) \geq \lambda \text { for } v \in \tau \cap \sigma, \\
& \left.t_{v}(x) \leq \lambda \text { for } v \notin \tau \cup \sigma\right\} \text {. }
\end{aligned}
$$

In particular

$$
\operatorname{cl}\langle\sigma\rangle_{\lambda} \cap \operatorname{cl}\langle\tau\rangle_{\lambda} \subseteq \operatorname{cl}\langle\sigma \cap \tau\rangle_{\lambda}
$$

Proof The proposition follows immediately from (11).

For $\lambda \in[0,1)$ let

$$
\varphi_{\lambda}:[0,1] \ni t \longmapsto\left\{\begin{array}{cc}
\frac{t-\lambda}{1-\lambda} & \text { for } t \geq \lambda \\
0 & \text { for } t \leq \lambda
\end{array} \in[0,1]\right.
$$

Given a simplex $\sigma$ in $\mathcal{X}$, we define the map

$$
\varphi_{\sigma}^{\lambda}: \operatorname{cl}\langle\sigma\rangle_{\lambda} \ni x \longmapsto \varphi_{\sigma}^{\lambda}(x) \in|\sigma|
$$

by

$$
\varphi_{\sigma}^{\lambda}(x):=\sum_{v \in \mathcal{X}_{0}} \frac{\varphi_{\lambda}\left(t_{v}(x)\right)}{\sum_{w \in \mathcal{X}_{0}} \varphi_{\lambda}\left(t_{w}(x)\right)} v .
$$

Proposition 8.3 The map $\varphi_{\sigma}^{\lambda}$ is well defined and continuous.

Proof Let $x \in \operatorname{cl}\langle\sigma\rangle_{\lambda}$. Then, we have $t_{v}(x) \leq \lambda$ for $v \notin \sigma$, and consequently, the identity $\varphi_{\lambda}\left(t_{v}(x)\right)=0$ holds for $v \notin \sigma$. Hence, $\varphi_{\sigma}^{\lambda}(x) \in|\sigma|$, which means that $\varphi_{\sigma}^{\lambda}$ is well defined. The continuity of $\varphi_{\sigma}^{\lambda}(x)$ follows from the continuity of $\varphi_{\lambda}$ and the continuity of the barycentric coordinates.

For $\sigma \in \mathcal{X}$ let $n_{\sigma}$ denote the number of vertices in $\sigma$. For $x \in \operatorname{cl}\langle\sigma\rangle_{\lambda}$ set

$$
r_{\sigma}^{\lambda}(x):=\sum_{w \notin \sigma} t_{w}(x)
$$

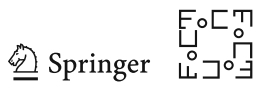


Lemma 8.4 Let $\sigma \in \mathcal{X}$ and $\lambda \in[0,1)$. For any $x \in \operatorname{cl}\langle\sigma\rangle_{\lambda}$ and $v \in \mathcal{X}_{0}$ we have

$$
t_{v}\left(\varphi_{\sigma}^{\lambda}(x)\right)=\left\{\begin{array}{cl}
\frac{t_{v}(x)-\lambda}{1-\lambda n_{\sigma}-r_{\sigma}^{\lambda}(x)} & \text { if } v \in \sigma \\
0 & \text { otherwise. }
\end{array}\right.
$$

Proof It is clear from (50) and (51) that (52) is correct for $v \notin \sigma$. On the other hand, if $v \in \sigma$, then $t_{v}(x) \geq \lambda$; hence, by (51) and (50) we have

$$
t_{v}(x)=t_{v}\left(\varphi_{\sigma}^{\lambda}(x)\right)(1-\lambda) \sum_{w \in \mathcal{X}_{0}} \varphi_{\lambda}\left(t_{w}(x)\right)+\lambda .
$$

Summing up the barycentric coordinates of $x$ over all vertices in $\mathcal{X}_{0}$, and taking into account the above equalities, which are valid for all vertices of $\sigma$, we obtain

$$
\begin{aligned}
\sum_{v \in \mathcal{X}_{0}} t_{v}(x) & =\sum_{v \in \sigma} t_{v}(x)+\sum_{v \notin \sigma} t_{v}(x) \\
& =\sum_{v \in \sigma} t_{v}\left(\varphi_{\sigma}^{\lambda}(x)\right)(1-\lambda) \sum_{w \in \mathcal{X}_{0}} \varphi_{\lambda}\left(t_{w}(x)\right)+\lambda n_{\sigma}+r_{\sigma}^{\lambda}(x) .
\end{aligned}
$$

Since the barycentric coordinates sum to 1 , we have $\sum_{v \in \mathcal{X}_{0}} t_{v}(x)=1$. Moreover, since $\varphi_{\sigma}^{\lambda}(x) \in|\sigma|$, we also have $\sum_{v \in \sigma} t_{v}\left(\varphi_{\sigma}^{\lambda}(x)\right)=1$. Therefore, the above equality reduces to

$$
1=(1-\lambda) \sum_{w \in \mathcal{X}_{0}} \varphi_{\lambda}\left(t_{w}(x)\right)+\lambda n_{\sigma}+r_{\sigma}^{\lambda}(x) .
$$

Consequently,

$$
\sum_{w \in \mathcal{X}_{0}} \varphi_{\lambda}\left(t_{w}(x)\right)=\frac{1-\lambda n_{\sigma}-r_{\sigma}^{\lambda}(x)}{1-\lambda} .
$$

Replacing the sum $\sum_{w \in \mathcal{X}_{0}} \varphi_{\lambda}\left(t_{w}(x)\right)$ in (53) by the right-hand side of this equation and calculating $t_{v}\left(\varphi_{\sigma}^{\lambda}(x)\right)$, we obtain (52) for $v \in \sigma$. This completes the proof.

Proposition 8.5 For any simplex $\sigma \in \mathcal{X}$ and $\lambda \in[0,1)$, we have

$$
\varphi_{\sigma}^{\lambda}\left(\sigma \cap \operatorname{cl}\langle\sigma\rangle_{\lambda}\right)=\sigma
$$

Proof It is clear that $\varphi_{\sigma}^{\lambda}\left(\sigma \cap \operatorname{cl}\langle\sigma\rangle_{\lambda}\right) \subseteq \sigma$,; therefore, we verify the opposite inclusion. Take an arbitrary $y \in \sigma$ and define

$$
x:=\sum_{v \in \sigma}\left(t_{v}(y)\left(1-\lambda n_{\sigma}\right)+\lambda\right) v
$$


It is easy to check that the above formula correctly defines a point $x \in \sigma$ via its barycentric coordinates. Moreover, we have $x \in \sigma \cap \operatorname{cl}\langle\sigma\rangle_{\lambda}$, as $t_{v}(x)=0$ for $v \notin \sigma$ and $t_{v}(x) \geq \lambda$ for $v \in \sigma$. An easy calculation, with the use of Lemma 8.4, finally shows that $\varphi_{\sigma}^{\lambda}(x)=y$.

The following proposition is an immediate consequence of Proposition 8.2.

Proposition 8.6 For any simplices $\sigma$ and $\tau$ in $\mathcal{X}$ and arbitrary $\lambda \in[0,1)$, the maps $\varphi_{\sigma}^{\lambda}$ and $\varphi_{\tau}^{\lambda}$ coincide on $\operatorname{cl}\langle\sigma\rangle_{\lambda} \cap \operatorname{cl}\langle\tau\rangle_{\lambda}$.

\subsection{Mapping $\psi$}

In view of (12) and Proposition 8.6, we have a well-defined continuous surjection $\varphi:|\mathcal{X}| \rightarrow|\mathcal{X}|$ given by

$$
\varphi(x):=\varphi_{\sigma}^{\delta}(x) \quad \text { where } \sigma \in \mathcal{X} \text { is such that } \quad x \in \operatorname{cl}\langle\sigma\rangle_{\lambda}
$$

Let $\psi:=\varphi_{\mid Q_{1}}: Q_{1} \rightarrow X$ denote the restriction of $\varphi$ to $Q_{1}$.

Proposition 8.7 For each $y \in|\mathrm{Cl} \mathcal{S}|$, the fiber $\psi^{-1}(y)$ is non-empty and contractible.

Proof Let $y \in|\mathrm{Cl} \mathcal{S}|=\langle\mathrm{Cl} \mathcal{S}\rangle$ be arbitrary and let the simplex $\sigma \in \mathrm{Cl} \mathcal{S}$ be such that $y \in \stackrel{\circ}{\sigma}$. Furthermore, define the set

$$
\begin{aligned}
X_{\sigma}:=\left\{x \in X \mid t_{v}(x)\right. & \leq \delta \text { if } v \notin \sigma \text { and } \\
t_{v}(x) & \left.=t_{v}(y)\left(1-\delta n_{\sigma}-r_{\sigma}^{\lambda}(x)\right)+\delta \text { for } v \in \sigma\right\} .
\end{aligned}
$$

We first verify that the fiber of $y$ under $\varphi_{\sigma}^{\delta}$ is given by

$$
\left(\varphi_{\sigma}^{\delta}\right)^{-1}(y)=X_{\sigma}
$$

For this, fix an $x \in X_{\sigma}$, and recall that $\delta$ satisfies (19), in particular $\delta<1 /(d+1)$. Therefore, for $v \in \sigma$ we deduce from (52) the inequality

$$
t_{v}(x)=t_{v}(y)\left(1-\delta n_{\sigma}-r_{\sigma}^{\lambda}(x)\right)+\delta \geq t_{v}(y)\left(1-\delta n_{\sigma}-\delta\left(d+1-n_{\sigma}\right)+\delta \geq \delta .\right.
$$

This, together with the obvious inequality $t_{v}(x) \leq \delta$ for $v \notin \sigma$, shows that

$$
X_{\sigma} \subseteq \operatorname{cl}\langle\sigma\rangle_{\delta}=\operatorname{dom} \varphi_{\sigma}^{\delta}
$$

Moreover, a straightforward calculation implies that for every point $x \in X_{\sigma}$ the identity $\varphi_{\sigma}^{\delta}(x)=y$ holds. This shows that $X_{\sigma} \subseteq\left(\varphi_{\sigma}^{\delta}\right)^{-1}(y)$. Since the converse inclusion is straightforward, the proof of (54) is finished. Now let

$$
\bar{X}_{\sigma}:=\left(\varphi_{\sigma}^{\delta}\right)^{-1}(y) \cap N_{\delta^{\prime}}(\mathrm{Cl} \mathcal{S})=X_{\sigma} \cap N_{\delta^{\prime}}(\mathrm{Cl} \mathcal{S}) .
$$

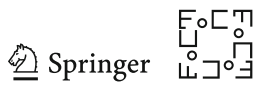


We claim that

$$
\psi^{-1}(y)=\bar{X}_{\sigma}
$$

Note that

$$
\bar{X}_{\sigma} \subseteq \operatorname{cl}\langle\sigma\rangle_{\delta} \cap N_{\delta^{\prime}}(\mathrm{Cl} \mathcal{S}) \subseteq N_{\delta} \cap N_{\delta^{\prime}}=Q_{1}=\operatorname{dom} \psi .
$$

Since $\bar{X}_{\sigma} \subseteq\left(\varphi_{\sigma}^{\delta}\right)^{-1}(y)$, one obtains for $w \in \bar{X}_{\sigma}$ the identity $y=\varphi_{\sigma}^{\delta}(w)=\psi(w)$. Therefore, $\bar{X}_{\sigma} \subseteq \psi^{-1}(y)$. For the proof of the reverse inclusion, it suffices to verify that the condition $x \notin \operatorname{cl}\langle\sigma\rangle_{\delta}$ implies $\psi(x) \neq y$. Suppose to the contrary that $x \notin \operatorname{cl}\langle\sigma\rangle_{\delta}$ and $\psi(x)=y$, and consider a simplex $\tau$ such that $x \in \operatorname{cl}\langle\tau\rangle_{\delta}$. Then, $\psi(x)=\varphi_{\tau}^{\delta}(x)$. Directly from the definition of $\varphi_{\tau}^{\delta}$, we infer that $\psi(x) \in \tau$. However, $\psi(x)=y \in \stackrel{\circ}{\sigma}$, which means that $\sigma$ is a face of $\tau$. Then, taking into account the inclusion $x \in \operatorname{cl}\langle\tau\rangle_{\delta} \backslash$ $\operatorname{cl}\langle\sigma\rangle_{\delta}$, we can find a vertex $v \in \tau \backslash \sigma$ such that $t_{v}(x)>\delta$. Consequently, by (52), we have $t_{v}(y)=t_{v}(\psi(x))=t_{v}\left(\varphi_{\tau}^{\delta}(x)\right)>0$, which contradicts $y \in \stackrel{\circ}{\sigma}$, and completes the proof of (57).

We still need to show that $\bar{X}_{\sigma}=\psi^{-1}(y)$ is contractible. To this end, we define the $\operatorname{map} h: \bar{X}_{\sigma} \times[0,1] \rightarrow \bar{X}_{\sigma}$ by

$$
h(x, s):=\sum_{v \in \mathcal{X}_{0}} t_{v, x, s} v
$$

where

$$
t_{v, x, s}:= \begin{cases}(1-s) t_{v}(x) & \text { if } v \notin \sigma \\ t_{v}(y)\left(1-\delta n_{\sigma}-(1-s) r_{\sigma}^{\lambda}(x)\right)+\delta & \text { if } v \in \sigma\end{cases}
$$

We will show that $h$ is a well-defined homotopy between the identity on $\bar{X}_{\sigma}$ and a constant map on $\bar{X}_{\sigma}$.

To begin with, we verify that for any point $x \in \bar{X}_{\sigma}$ and arbitrary $s \in[0,1]$ we have $h(x, s) \in \bar{X}_{\sigma}$. The verification that the inclusion $h(x, s) \in \operatorname{cl}\langle\sigma\rangle_{\delta}$ holds, as well as $\varphi_{\sigma}^{\delta}(h(x, s))=y$, which in turn shows that $h(x, s) \in\left(\varphi_{\sigma}^{\delta}\right)^{-1}(y)$, is tedious but straightforward. We still need to verify that $h(x, s) \in N_{\delta^{\prime}}(\mathrm{Cl} \mathcal{S})$. For this, consider a simplex $\tau \in \mathrm{Cl} \mathcal{S}$ such that $x \in \operatorname{cl}\langle\sigma\rangle_{\delta} \cap \operatorname{cl}\langle\tau\rangle_{\delta^{\prime}}$. Since for any $v \in \sigma$ we have $t_{v}(x) \geq \delta>\delta^{\prime}$, we deduce that $\sigma \subseteq \tau$. Let

$$
\eta:=\left\{v \in \mathcal{X}_{0} \mid t_{v}(h(x, s))>\delta^{\prime}\right\}
$$

Then, we claim that the inclusions $\sigma \subseteq \eta \subseteq \tau$ hold. Indeed, if $v \notin \tau$, then $\sigma \subseteq \tau$ implies the inequalities $t_{v}(h(x, s))=(1-s) t_{v}(x) \leq t_{v}(x) \leq \delta^{\prime}$. Furthermore, if one has $v \in \sigma \subseteq \tau$, then $t_{v}(h(x, s)) \geq t_{v}(x) \geq \delta>\delta^{\prime}$. Therefore, $\eta$ is a simplex and it satisfies $h(x, s) \in \operatorname{cl}\langle\eta\rangle_{\delta^{\prime}}$. Since $\sigma \in \mathrm{Cl} \mathcal{S}$ as well as $\tau \in \mathrm{Cl} \mathcal{S}$, the closedness of $\mathrm{Cl} \mathcal{S}$ implies that $\eta \in \mathrm{Cl} \mathcal{S}$. Consequently, $h(x, s) \in N_{\delta^{\prime}}(\mathrm{Cl} \mathcal{S})$, and this proves that the map $h$ is well defined.

The continuity of $h$ follows from the continuity of the barycentric coordinates. Verification that $h(\cdot, 0)=\mathrm{id}_{\bar{X}_{\sigma}}$ as well as that $h(\cdot, 1)$ is constant on $\bar{X}_{\sigma}$ is straightforward. This completes the proof. 
Proposition 8.8 We have

(i) $\psi\left(Q_{1}\right)=|\mathrm{Cl} \mathcal{S}|$,

(ii) $\psi\left(Q_{2}\right)=|\operatorname{Ex} \mathcal{S}|$,

(iii) $\psi^{-1}(|\operatorname{Ex} \mathcal{S}|)=Q_{2}$.

In particular, we can consider $\psi$ as a map of pairs

$$
\psi:\left(Q_{1}, Q_{2}\right) \rightarrow(|\mathrm{Cl} \mathcal{S}|,|\operatorname{Ex} \mathcal{S}|)
$$

Proof For the proof of (i), fix an arbitrary point $x \in Q_{1}$. Then, there exists a simplex $\sigma \in \mathrm{Cl} \mathcal{S}$ with $x \in \operatorname{cl}\langle\sigma\rangle_{\delta}$. Thus, $\psi(x)=\varphi(x)=\varphi_{\sigma}^{\delta}(x) \in \sigma \subseteq|\mathrm{Cl} \mathcal{S}|$ and $\psi(x) \in|\mathrm{Cl} \mathcal{S}|$. This implies that the inclusion $\psi\left(Q_{1}\right) \subseteq|\mathrm{Cl} \mathcal{S}|$. The reverse inclusion is a consequence of Proposition 8.5 because for any simplex $\sigma \in \mathrm{Cl} \mathcal{S}$ we have $\sigma \cap \operatorname{cl}\langle\sigma\rangle_{\delta} \subseteq \operatorname{cl}\langle\sigma\rangle_{\delta} \cap \operatorname{cl}\langle\sigma\rangle_{\delta^{\prime}} \subseteq Q_{1}$. The proof of (ii) is analogous to the proof of (i).

In order to prove the remaining statement (iii), first observe that we have the inclusion $\psi^{-1}(|\operatorname{Ex} \mathcal{S}|) \subseteq Q_{2}$. Indeed, given a $y \in|\operatorname{Ex} \mathcal{S}|$, there exists a $\sigma \in \operatorname{Ex} \mathcal{S}$ such that $y \in \stackrel{\circ}{\circ}$, and by (56), (57), and (55), we have

$$
\psi^{-1}(y) \subseteq \operatorname{cl}\langle\sigma\rangle_{\delta} \cap N_{\delta^{\prime}}(\mathrm{Cl} \mathcal{S}) \subseteq N_{\delta}(\operatorname{Ex} \mathcal{S}) \cap N_{\delta^{\prime}}(\mathrm{Cl} \mathcal{S})=Q_{2}
$$

which implies $\psi^{-1}(|\operatorname{Ex} \mathcal{S}|) \subseteq Q_{2}$. This, together with (ii), implies (iii). The last statement is a direct consequence of (i) and (ii).

Proposition 8.9 We have

$$
H^{*}\left(Q_{1}, Q_{2}\right) \cong H^{*}(|\mathrm{Cl} \mathcal{S}|,|\mathrm{Ex} \mathcal{S}|)
$$

Proof By Proposition 8.8 the mapping $\psi:\left(Q_{1}, Q_{2}\right) \rightarrow(|\mathrm{Cl} \mathcal{S}|,|\mathrm{Ex} \mathcal{S}|)$ is a continuous surjection with $\psi^{-1}(|\operatorname{Ex} \mathcal{S}|)=Q_{2}$. By Proposition 8.7, $\psi$ has contractible, and hence acyclic fibers. Moreover, $\psi$ is proper, that is, the preimages of compact sets under $\psi$ are compact. Therefore, the map $\psi$ is a Vietoris map. By the Vietoris-Begle mapping theorem for the pair of spaces, we conclude that

$$
\psi^{*}: H^{*}(|\mathrm{Cl} \mathcal{S}|,|\operatorname{Ex} \mathcal{S}|) \rightarrow H^{*}\left(Q_{1}, Q_{2}\right)
$$

is an isomorphism, which completes the proof.

Figure 13 shows an example of the pairs $\left(Q_{1}, Q_{2}\right)$ and $(|\mathrm{Cl} \mathcal{S}|,|\mathrm{Ex} \mathcal{S}|)$ in Proposition 8.9 .

Proof of Theorem 5.4: Theorem 5.4 is an immediate consequence of Propositions 8.1, 8.9, and Theorem 5.3.

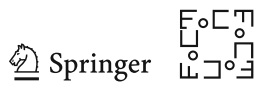




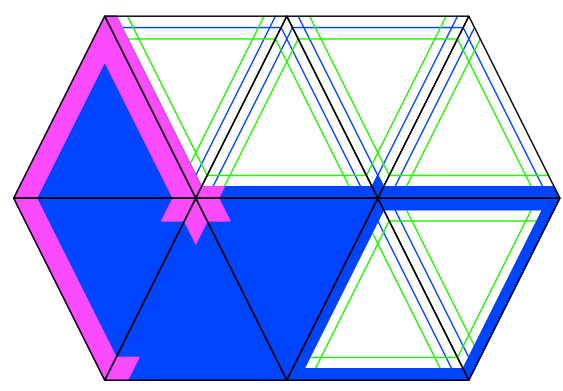

(A) The pair $Q=\left(Q_{1}, Q_{2}\right)$. $Q_{1}$ is the union of the dark blue and magenta regions, while $Q_{2}$ is only the magenta region.

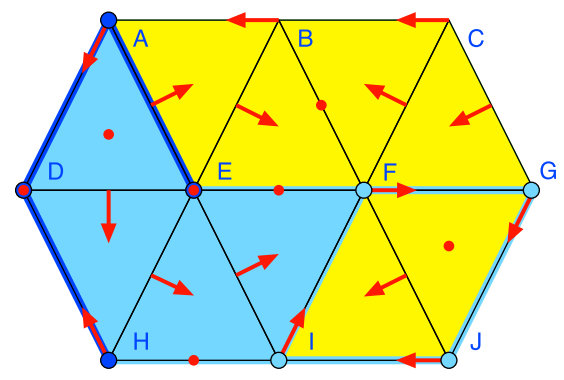

(B) The pair $(|\mathrm{Cl} \mathcal{S}|,|\operatorname{Ex} \mathcal{S}|) . \quad|\mathrm{Cl} \mathcal{S}|$ is the union of the light blue and dark blue regions, while $|\operatorname{Ex} \mathcal{S}|$ is only the dark blue set.

Fig. 13 The pairs $Q=\left(Q_{1}, Q_{2}\right)$ and $(|\mathrm{Cl} \mathcal{S}|,|\operatorname{Ex} \mathcal{S}|)$

\section{Proof of Theorem 5.5}

In order to prove Theorem 5.5, we first establish a few auxiliary lemmas. Then, we recall some results concerning the correspondence of solutions for $\Pi_{\mathcal{V}}$ and $F$. We then use this correspondence to prove an auxiliary theorem and finally present the proof of Theorem 5.5.

\subsection{Auxiliary Lemmas}

First observe that Theorem 5.1 applies to the set $N_{\beta}(\mathcal{S})$ given by (17) for any $\beta$ which satisfies $0<\beta<1 /(d+1)$.

Lemma 9.1 We have

$$
N_{\varepsilon} \cap\langle\mathcal{S}\rangle=N_{\varepsilon} \cap|\mathrm{Cl} \mathcal{S}|
$$

In particular, $N_{\varepsilon} \cap\langle\mathcal{S}\rangle$ is closed.

Proof Clearly, $N_{\varepsilon} \cap\langle\mathcal{S}\rangle \subseteq N_{\varepsilon} \cap|\mathrm{Cl} \mathcal{S}|$. To prove the opposite inclusion, assume to the contrary that there exists an $x \in N_{\varepsilon} \cap|\mathrm{Cl} \mathcal{S}|$ and $x \notin N_{\varepsilon} \cap\langle\mathcal{S}\rangle$. Then, by Proposition 3.1(ii), $x \in N_{\varepsilon} \cap \mid$ Ex $\mathcal{S} \mid$. Consider simplices $\sigma \in \operatorname{Ex} \mathcal{S}$ and $\tau \in \mathcal{S}$ such that $x \in \stackrel{\circ}{\sigma}$ and $x \in \operatorname{cl}\langle\tau\rangle_{\varepsilon}$. Since for any vertex $v \in \tau$ we have $t_{v}(x) \geq \varepsilon>0$, the inclusion $v \in \sigma$ has to hold. Hence, $\tau \subseteq \sigma$. Therefore, by the closedness of Ex $\mathcal{S}$ we get $\tau \in \operatorname{Ex} \mathcal{S}$, a contradiction.

Lemma 9.2 For any $x \in N_{\varepsilon} \cap\langle\mathcal{S}\rangle$ we have $\sigma_{\max }^{\varepsilon}(x) \in \mathcal{S}$.

Proof Fix a point $x \in N_{\varepsilon} \cap\langle\mathcal{S}\rangle$. Then, there exist simplices $\tau, \sigma \in \mathcal{S}$ such that $x \in \operatorname{cl}\langle\tau\rangle_{\varepsilon}$ and $x \in \stackrel{\circ}{\sigma}$. Clearly, one has $\sigma=\sigma_{\text {min }}^{0}(x)$. By Corollary 4.4 and Lemma 4.1, we then obtain $\tau \subseteq \sigma_{\max }^{\varepsilon}(x) \subseteq \sigma$. Therefore, the closedness of Ex $\mathcal{S}$ implies that $\sigma_{\text {max }}^{\varepsilon}(x) \in \mathcal{S}$. 
Lemma 9.3 We have

$$
N_{\varepsilon} \cap\langle\mathcal{S}\rangle \subseteq N_{\delta} \cap\langle\mathcal{S}\rangle
$$

Proof Fix a point $x \in N_{\varepsilon} \cap\langle\mathcal{S}\rangle$. Then, we have $\sigma_{\text {min }}^{0}(x) \in \mathcal{S}$. By Lemma 9.2 we further obtain $\sigma_{\max }^{\varepsilon}(x) \in \mathcal{S}$. In addition, Lemma 4.1 immediately implies the inclusions $\sigma_{\max }^{\varepsilon}(x) \subseteq \sigma_{\min }^{\delta}(x) \subseteq \sigma_{\max }^{\delta}(x) \subseteq \sigma_{\min }^{0}(x)$. Now the closedness of Ex $\mathcal{S}$ yields $\sigma_{\max }^{\delta}(x) \in \mathcal{S}$, and consequently, $x \in N_{\delta}$, which completes the proof.

\subsection{Solution Correspondence}

In the sequel we need two results on the correspondence of solutions of the combinatorial flow $\Pi_{\mathcal{V}}$ and the associated multivalued dynamical system $F$. We recall them from [19]. We begin with a definition.

Definition 9.4 (see [19, Definition 5.2])

(a) Let $\varrho: \mathbb{Z} \rightarrow \mathcal{X}$ denote a full solution of the combinatorial flow $\Pi_{\mathcal{V}}$. Then, the reduced solution $\varrho^{*}: \mathbb{Z} \rightarrow \mathcal{X}$ is obtained from $\varrho$ by removing $\varrho(k+1)$ whenever $\varrho(k+1)$ is the target of an arrow of $\mathcal{V}$ whose source is $\varrho(k)$.

(b) Conversely, let $\varrho^{*}: \mathbb{Z} \rightarrow \mathcal{X}$ denote an arbitrary sequence of simplices in $\mathcal{X}$. Then, its arrowhead extension $\varrho: \mathbb{Z} \rightarrow \mathcal{X}$ is defined as follows. If $\varrho^{*}(k) \in \operatorname{dom} \mathcal{V} \backslash$ Fix $\mathcal{V}$ and if $\varrho^{*}(k+1) \neq \varrho^{*}(k)^{+}$, then we insert $\varrho^{*}(k)^{+}$between $\varrho^{*}(k)$ and $\varrho^{*}(k+1)$. In other words, the arrowhead extension $\varrho$ is obtained from $\varrho^{*}$ by inserting missing targets of arrows.

Theorem 9.5 (see [19, Theorem 5.3]) Let

$$
X^{\varepsilon}:=\bigcup_{\sigma \in \mathcal{X}}\langle\sigma\rangle_{\varepsilon} \subseteq X
$$

denote the union of all open $\varepsilon$-cells of $X$. Then, the following holds.

(a) Let $\varrho: \mathbb{Z} \rightarrow \mathcal{X}$ denote a full solution of the combinatorial flow $\Pi_{\mathcal{V}}$. Furthermore, let $\varrho^{*}: \mathbb{Z} \rightarrow \mathcal{X}$ denote the reduced solution as in Definition 9.4(a). Then, there is a function $\varphi: \mathbb{Z} \rightarrow X^{\varepsilon}$ such that for $k \in \mathbb{Z}$ we have

$$
\varphi(k+1) \in F(\varphi(k)) \text { and } \varphi(k) \in\left\langle\varrho^{*}(k)\right\rangle_{\varepsilon} .
$$

In other words, $\varphi$ is an orbit of $F$ which follows the dynamics of the combinatorial simplicial solution $\varrho$ after removing arrowheads.

(b) Conversely, let $\varphi: \mathbb{Z} \rightarrow X^{\varepsilon}$ denote a full solution of $F$ which is completely contained in $X^{\varepsilon}$. Let $\varrho^{*}(k)=\sigma_{\max }^{\varepsilon}(\varphi(k))$ for $k \in \mathbb{Z}$, and let $\varrho: \mathbb{Z} \rightarrow \mathcal{X}$ denote the arrowhead extension of $\varrho$ as in Definition 9.4(b). Then, $\varrho$ is a solution of the combinatorial flow $\Pi_{\mathcal{V}}$. 
Lemma 9.6 (see [19, Lemma 4.9]) For all simplices $\sigma \in \mathcal{X}$ and all points $x \in X$, we have $F_{\sigma}(x) \subseteq \sigma^{+}$.

Theorem 9.7 (see [19, Theorem 5.4]) Let $\varphi: \mathbb{Z} \rightarrow X$ denote an arbitrary full solution of the multivalued map $F$ and let

$$
\varrho^{*}(k)=\sigma_{\max }^{\varepsilon}(\varphi(k))
$$

for $k \in \mathbb{Z}$. Extend this sequence of simplices in the following way:

(1) For all $k \in \mathbb{Z}$ with $\varphi(k) \notin\left|\varrho^{*}(k-1)^{+}\right|$, we choose a face $\tau \subseteq \varrho^{*}(k-1)$ such that $\varphi(k) \in\left|\mathrm{Cl} \tau^{+} \backslash\{\tau\}\right|$ and then insert $\tau$ between $\varrho^{*}(k-1)$ and $\varrho^{*}(k)$.

(2) Let $\varrho: \mathbb{Z} \rightarrow \mathcal{X}$ denote the arrowhead extension of the sequence created in (1), according to Definition $9.4(b)$.

Then, the so-obtained simplex sequence $\varrho: \mathbb{Z} \rightarrow \mathcal{X}$ is a solution of the combinatorial flow $\Pi_{\mathcal{V}}$.

\subsection{Invariance}

Lemma 9.8 The set $N_{\varepsilon} \cap\langle\mathcal{S}\rangle$ is negatively invariant with respect to $F$, that is

$$
\operatorname{Inv}_{F}^{-}\left(N_{\varepsilon} \cap\langle\mathcal{S}\rangle\right)=N_{\varepsilon} \cap\langle\mathcal{S}\rangle .
$$

Proof Obviously, it suffices to prove that for every $y \in N_{\varepsilon} \cap\langle\mathcal{S}\rangle$ there exists an $x \in N_{\varepsilon} \cap\langle\mathcal{S}\rangle$ such that $y \in F(x)$. To verify this, fix a $y \in N_{\varepsilon} \cap\langle\mathcal{S}\rangle$. Let $\sigma \in \mathcal{S}$ be such that $y \in \stackrel{\circ}{\sigma}$. We will consider several cases concerning the simplex $\sigma$. First assume that $\sigma \in$ Fix $\mathcal{V}$, that is, $\sigma=\sigma^{-}=\sigma^{+}$. Take any $x \in\langle\sigma\rangle_{\varepsilon} \cap \sigma \subseteq N_{\varepsilon} \cap\langle\mathcal{S}\rangle$. Since $\sigma_{\max }^{\varepsilon}(x)=\sigma^{-}=\sigma^{+}$, the definition of $F$ (see (16)) shows that $F_{\sigma}(x)=\sigma$. Hence, $y \in F_{\sigma}(x) \subseteq F(x)$.

Now assume that $\sigma^{-} \neq \sigma^{+}$. Note that if $\sigma=\sigma^{+}$, then we have $\sigma=A_{\sigma} \cup B_{\sigma}$, and if $\sigma=\sigma^{-}$, then $\sigma \subseteq A_{\sigma}$. Hence, either $y \in A_{\sigma}$ or $\sigma=\sigma^{+}$and $y \in B_{\sigma}$. In the latter case we may take any point $x \in\langle\sigma\rangle_{\varepsilon} \cap \sigma \subseteq N_{\varepsilon} \cap\langle\mathcal{S}\rangle$ because in that case one has $\sigma=\sigma_{\max }^{\varepsilon}(x)=\sigma_{\max }^{\varepsilon}(x)^{+} \neq \sigma_{\max }^{\varepsilon}(x)^{-}$, which immediately yields the inclusion $y \in B_{\sigma}=F_{\sigma}(x) \subseteq F(x)$.

It remains to consider the case $y \in A_{\sigma}$. Since $\mathcal{S}$ is invariant with respect to $\Pi_{\mathcal{V}}$ and $\sigma^{+}=\mathcal{V}\left(\sigma^{-}\right)$, there exists a trajectory $\varrho$ of $\mathcal{V}$ in $\mathcal{S}$ which contains $\sigma^{-}$and $\sigma^{+}$as consecutive simplices. Let $\tau$ denote the simplex in this solution which precedes the tail $\sigma^{-} \in \operatorname{dom} \mathcal{V}$. Then, $\tau \in \mathcal{S}$ and, according to the definition of the multivalued flow $\Pi_{\mathcal{V}}$, we have $\sigma^{-} \subsetneq \tau \neq \sigma^{+}$. Now let $k$ denote the number of vertices in $\tau \backslash \sigma$ and let $x \in X$ be the point with the barycentric coordinates given by

$$
t_{v}(x):= \begin{cases}\varepsilon & \text { if } v \in \tau \backslash \sigma \\ 1-k \varepsilon & \text { if } v \in \sigma \\ 0 & \text { otherwise. }\end{cases}
$$


Then, we have both $x \in \operatorname{cl}\langle\tau\rangle_{\varepsilon} \cap \operatorname{cl}\left\langle\sigma^{-}\right\rangle_{\varepsilon} \cap \tau$ and $\sigma_{\max }^{\varepsilon}(x)=\tau$, and this in turn implies $\sigma_{\max }^{\varepsilon}(x)^{+} \neq \sigma^{-}$and $\sigma_{\max }^{\varepsilon}(x)^{-} \neq \sigma^{-}$. Therefore, $F_{\sigma^{-}}(x)=A_{\sigma^{-}}=A_{\sigma}$, which shows that $y \in F_{\sigma^{-}}(x) \subseteq F(x)$.

Lemma 9.9 The set $N_{\varepsilon} \cap\langle\mathcal{S}\rangle$ is positively invariant with respect to $F$, that is

$$
\operatorname{Inv}_{F}^{+}\left(N_{\varepsilon} \cap\langle\mathcal{S}\rangle\right)=N_{\varepsilon} \cap\langle\mathcal{S}\rangle .
$$

Proof For the proof it is enough to justify that for any point $x \in N_{\varepsilon} \cap\langle\mathcal{S}\rangle$ we have $F(x) \cap N_{\varepsilon} \cap\langle\mathcal{S}\rangle \neq \varnothing$. Let $x \in N_{\varepsilon} \cap\langle\mathcal{S}\rangle$ be fixed and let $\sigma:=\sigma_{\max }^{\varepsilon}(x)$. By Lemma 9.2 we have $\sigma \in \mathcal{S}$. Then, $x \in \operatorname{cl}\langle\sigma\rangle_{\varepsilon}$. Since $F\left(\langle\sigma\rangle_{\varepsilon}\right) \subseteq F\left(\operatorname{cl}\langle\sigma\rangle_{\varepsilon}\right)$ and $F$ is strongly upper semicontinuous by Theorem 4.6, without loss of generality we may assume that $x \in\langle\sigma\rangle_{\varepsilon}$. The set $\mathcal{S}$ is invariant with respect to the combinatorial flow $\Pi_{\mathcal{V}}$. Hence, there exists a solution $\varrho: \mathbb{Z} \rightarrow \mathcal{X}$ of $\Pi_{\mathcal{V}}$, which is contained in $\mathcal{S}$ and passes through $\sigma$. Furthermore, let $\varrho^{*}: \mathbb{Z} \rightarrow \mathcal{X}$ denote the reduced solution as defined in Definition 9.4(a). There are two possible complementary cases: $\sigma \in \operatorname{im} \varrho^{*}$ or $\sigma \notin \operatorname{im} \varrho^{*}$.

In the first case there exists a $k \in \mathbb{Z}$ with $\sigma=\varrho^{*}(k)$. Consider $\varphi: \mathbb{Z} \rightarrow \mathcal{X}^{\varepsilon}$, which is a corresponding solution with respect to $F$ as constructed in Theorem 9.5(a). Then, $\varphi(k) \in\langle\sigma\rangle_{\varepsilon}$ and $\varphi(k+1) \in F(\varphi(k)) \cap\left\langle\varrho^{*}(k+1)\right\rangle_{\varepsilon}$. Since the map $F$ is constant on open $\varepsilon$-cells, we further obtain

$$
\varphi(k+1) \in F(x) \cap\left\langle\varrho^{*}(k+1)\right\rangle_{\varepsilon} .
$$

Due to $x \in\langle\sigma\rangle_{\varepsilon}$, by Lemma 9.6 we have $F(x)=F_{\sigma}(x) \subseteq \sigma^{+} \in \mathcal{S}$, where the last inclusion follows from Proposition 3.4, as $\mathcal{S}$ is an isolated invariant set. This, along with (59), completes the proof in the case where $\sigma \in \operatorname{im} \varrho^{*}$.

Finally, we consider the case $\varrho(k):=\sigma \notin \operatorname{im} \varrho^{*}$, which immediately gives rise to the inclusion $\sigma \in \operatorname{im} \mathcal{V} \backslash$ Fix $\mathcal{V}$. In this case, the identity $\Pi_{\mathcal{V}}(\sigma)=\operatorname{Ex} \sigma \backslash\left\{\sigma^{-}\right\}$implies $\varrho(k+1) \in \operatorname{Ex} \sigma \backslash\left\{\sigma^{-}\right\}$. However, we also have $F(x)=F_{\sigma}(x)=B_{\sigma}$, according to the fact that $\sigma=\sigma_{\max }^{\varepsilon}(x)^{+} \neq \sigma_{\max }^{\varepsilon}(x)^{-}$. This readily furnishes the inclusions $|\varrho(k+1)| \subseteq\left|\operatorname{Ex} \sigma \backslash\left\{\sigma^{-}\right\}\right| \subseteq B_{\sigma}=F(x)$. In particular, the barycenter of $\varrho(k+1)$ belongs to $|\varrho(k+1)| \cap\langle\varrho(k+1)\rangle_{\varepsilon} \cap F(x) \subseteq F(x) \cap N_{\varepsilon} \cap\langle\mathcal{S}\rangle$. This completes the proof.

As a straightforward consequence of Lemmas 9.8 and 9.9, we obtain the following corollary.

Corollary 9.10 The set $N_{\varepsilon} \cap\langle\mathcal{S}\rangle$ is invariant with respect to $F$, that is, we have

$$
\operatorname{Inv}_{F}\left(N_{\varepsilon} \cap\langle\mathcal{S}\rangle\right)=N_{\varepsilon} \cap\langle\mathcal{S}\rangle .
$$

\subsection{An Auxiliary Theorem and Lemma}

The following characterization of the set $S(\mathcal{S})=\operatorname{Inv} N_{\delta}$ is needed in the proof of Theorem 5.5.

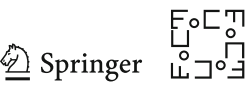


Theorem 9.11 We have

$$
\operatorname{Inv} N_{\delta}=N_{\varepsilon} \cap\langle\mathcal{S}\rangle .
$$

Proof According to Lemma 9.3 and Corollary 9.10, we immediately obtain the inclusion $N_{\varepsilon} \cap\langle\mathcal{S}\rangle \subseteq \operatorname{Inv}_{F} N_{\delta}$. Therefore, it suffices to verify the opposite inclusion.

To accomplish this, take an $x \in N_{\delta} \backslash\left(N_{\varepsilon} \cap\langle\mathcal{S}\rangle\right)$. If $x \in N_{\delta} \backslash N_{\varepsilon}$, then $x \in \operatorname{cl}\langle\tau\rangle_{\delta}$ for some simplex $\tau \in \mathcal{S}$, and $\sigma_{\text {max }}^{\varepsilon}(x) \notin \mathcal{S}$. This, according to Lemma 6.2, implies $F(x) \cap N_{\delta}=\varnothing$. If $x \in N_{\delta} \backslash\langle\mathcal{S}\rangle$, then we again obtain $F(x) \cap N_{\delta}=\varnothing$ as a consequence of Lemma 6.4. Both cases show that there is no solution with respect to $F$ passing through $x$ and contained in $N_{\delta}$, which means that $\operatorname{Inv}_{F} N_{\delta} \subseteq N_{\varepsilon} \cap\langle\mathcal{S}\rangle$, and therefore completes the proof.

Note that by Theorem 9.11 the sets $M_{r}$ can be alternatively expressed as

$$
M_{r}=\operatorname{Inv}_{F}\left(N_{\delta}^{r}\right),
$$

where $N_{\delta}^{r}=\bigcup_{\sigma \in \mathcal{M}_{r}} \operatorname{cl}\langle\sigma\rangle_{\delta}$.

Lemma 9.12 Let $\varphi: \mathbb{Z} \rightarrow X$ be a solution for the multivalued map $F$. Assume that the sequence of simplices $\varrho^{*}: \mathbb{Z} \rightarrow \mathcal{X}$ and $\varrho: \mathbb{Z} \rightarrow \mathcal{X}$ define a corresponding solution of the combinatorial flow $\Pi_{\mathcal{V}}$, as introduced in Theorem 9.7. If $\mathcal{S}$ is an isolated invariant set with respect to $\Pi_{\mathcal{V}}$, and if there exists a integer $k \in \mathbb{Z}$ such that $\varrho^{*}(k), \varrho^{*}(k+1) \in \mathcal{S}$, and if each simplex in the extended solution $\varrho$ between the simplices $\varrho^{*}(k)$ and $\varrho^{*}(k+1)$ belongs to $\mathcal{S}$, then $\varphi(k+1) \in\langle\mathcal{S}\rangle \cap N_{\varepsilon}$.

Proof Observe that by (58) we have the inclusion $\varphi(k+1) \in\left\langle\varrho^{*}(k+1)\right\rangle_{\varepsilon}$, and since $\varrho^{*}(k+1) \in \mathcal{S}$, we get $\varphi(k+1) \in N_{\varepsilon}$. We need to verify that $\varphi(k+1) \in\langle\mathcal{S}\rangle$. Let $\sigma_{i}=\sigma_{\max }^{\varepsilon}(\varphi(i))=\varrho^{*}(i)$ for $i \in \mathbb{Z}$. Then, we have to consider the following two complementary cases: $\varphi(k+1) \in \sigma_{k}^{+}$and $\varphi(k+1) \notin \sigma_{k}^{+}$.

The first case immediately shows that $\varphi(k+1) \in|\mathrm{Cl} \mathcal{S}|$, as $\sigma_{k} \in \mathcal{S}$ implies the inclusion $\sigma_{k}^{+} \in \mathcal{S}$ according to the assertion that $\mathcal{S}$ is an isolated invariant set (cf. Proposition 3.3). Since we also have $\varphi(k+1) \in N_{\varepsilon}$, the inclusion $\varphi(k+1) \in\langle\mathcal{S}\rangle$ follows from Lemma 9.1.

Consider now the second case $\varphi(k+1) \notin \sigma_{k}^{+}$. According to Theorem 9.7(1), we have the inclusion $\varphi(k+1) \in\left|\mathrm{Cl} \tau^{+}\right|$, where $\tau$ is a simplex in the extended solution $\varrho$ which lies between the simplices $\varrho^{*}(k)$ and $\varrho^{*}(k+1)$. According to our assumption $\tau$ belongs to $\mathcal{S}$, hence so does $\tau^{+}$, as $\mathcal{S}$ is isolated and invariant. Consequently, $\varphi(k+1) \in$ $|\mathrm{Cl} \mathcal{S}|$ which, along with the inclusion $\varphi(k+1) \in N_{\varepsilon}$ and Lemma 9.1, implies $\varphi(k+1) \in\langle\mathcal{S}\rangle$. This completes the proof.

\subsection{Proof of Theorem 5.5}

First note that the sets $M_{r}$ of $M$ are mutually disjoint, which is a consequence of the mutual disjointness of the sets in the family $\mathcal{M}$ and the definition of the sets $M_{r}$. Moreover, by Theorems 5.1 and 9.11, they are isolated invariant sets with respect to the map $F$. Hence, condition (a) of Definition 3.9 holds. 
We now verify condition (b) of Definition 3.9. Let $\varphi: \mathbb{Z} \rightarrow X$ be an arbitrary solution of the multivalued map $F$. Let $\varrho: \mathbb{Z} \rightarrow \mathcal{X}$ denote a corresponding solution of the multivalued flow $\Pi_{\mathcal{V}}$ as constructed in Theorem 9.7. Since $\mathcal{M}$ is a Morse decomposition of $\mathcal{V}$, there exist two indices $r, r^{\prime} \in \mathbb{P}$ with $r^{\prime} \geq r$, such that the inclusions $\alpha(\varrho) \subseteq \mathcal{M}_{r^{\prime}}$ and $\omega(\varrho) \subseteq \mathcal{M}_{r}$ are satisfied.

Let us first focus on the $\omega$-limit set of $\varphi$. The inclusion $\omega(\varrho) \subseteq \mathcal{M}_{r}$ implies that there exists a $k^{\prime} \in \mathbb{Z}^{+}$such that $\varrho(n) \in \mathcal{M}_{r}$ for all $n \geq k^{\prime}$. Passing to the reduced solution $\varrho^{*}$, we infer that $\varrho^{*}(n) \in \mathcal{M}_{r}$ for large enough $n$. Let $k \in \mathbb{Z}^{+}$be such that both $\varrho(n) \in \mathcal{M}_{r}$ and $\varrho^{*}(n) \in \mathcal{M}_{r}$ hold for $n \geq k$. Then, Lemma 9.12 implies the inclusion $\varphi(k+1) \in M_{r}$. Applying Lemma 9.12 another time, we further obtain the inclusion $\varphi([k+1,+\infty)) \subseteq M_{r}$, which in combination with the closedness of $M_{r}$ yields $\omega(\varphi) \subseteq M_{r}$.

For the set $\alpha(\varphi)$ a similar argument applies. Indeed, the inclusion $\alpha(\varrho) \subseteq \mathcal{M}_{r^{\prime}}$ establishes the existence of $k \in \mathbb{Z}^{+}$with $\varrho(-n) \in \mathcal{M}_{r^{\prime}}$ and $\varrho^{*}(-n) \in \mathcal{M}_{r^{\prime}}$ for all integers $n \geq k$. Applying Lemma 9.12, this time to the arguments $-(k+1)$ and $-k$, we can further deduce that $\varphi(-k) \in M_{r^{\prime}}$. Now, by the reverse recurrence and Lemma 9.12, we have $\varphi((-\infty,-k]) \subseteq M_{r}$, and the inclusion $\alpha(\varphi) \subseteq M_{r^{\prime}}$ follows.

Next, we verify condition (c) of Definition 3.9. Suppose that $\varphi$ is a full solution of $F$ such that $\alpha(\varphi) \cup \omega(\varphi) \subseteq M_{r}$ for some $r \in \mathbb{P}$. Consider the corresponding solution $\varrho: \mathbb{Z} \rightarrow \mathcal{X}$ of the multivalued flow $\Pi_{\mathcal{V}}$, as constructed in Theorem 9.7. Since $\mathcal{M}$ is a Morse decomposition with respect to $\Pi_{\mathcal{V}}$, we have $\alpha(\varrho) \subseteq \mathcal{M}_{r_{1}}$ and $\omega(\varrho) \subseteq \mathcal{M}_{r_{2}}$, for some $r_{1}, r_{2} \in \mathbb{P}$. Then, the argument used for the proof of condition (b) shows that the two inclusions $\alpha(\varphi) \subseteq M_{r_{1}}$ and $\omega(\varphi) \subseteq M_{r_{2}}$ are satisfied. This immediately yields $r_{1}=r_{2}=r$, as $M$ is a family of disjoint sets. Thus, we have $\alpha(\varrho) \cup \omega(\varrho) \subseteq \mathcal{M}_{r}$. Since $\mathcal{M}$ is a Morse decomposition, the inclusion im $\varrho \subseteq \mathcal{M}_{r}$ follows, and consequently $\varrho(k) \in \mathcal{M}_{r}$ and $\varrho^{*}(k) \in \mathcal{M}_{r}$ for all $k \in \mathbb{Z}$. Again, by the recurrent argument with respect to $k$ in both forward and backward directions and Lemma 9.12, we conclude that $\operatorname{im} \varphi \subseteq M_{r}$. This completes the proof that the collection $M$ is a Morse decomposition of $X$ with respect to $F$.

The Conley indices of $\mathcal{M}_{r}$ and $M_{r}$ coincide by Theorem 5.4. The fact that the Conley-Morse graphs coincide as well follows from Theorems 9.7 and 9.5(a) via an argument similar to the argument for condition (b) of Definition 9.4 and is left to the reader.

Open Access This article is licensed under a Creative Commons Attribution 4.0 International License, which permits use, sharing, adaptation, distribution and reproduction in any medium or format, as long as you give appropriate credit to the original author(s) and the source, provide a link to the Creative Commons licence, and indicate if changes were made. The images or other third party material in this article are included in the article's Creative Commons licence, unless indicated otherwise in a credit line to the material. If material is not included in the article's Creative Commons licence and your intended use is not permitted by statutory regulation or exceeds the permitted use, you will need to obtain permission directly from the copyright holder. To view a copy of this licence, visit http://creativecommons.org/licenses/by/4.0/.

\section{References}

1. P.S. Alexandrov. Diskrete Räume, Mathematiceskii Sbornik (N.S.) 2(1937), 501-518.

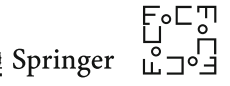


2. Z. Arai, W. Kalies, H. Kokubu, K. Mischaikow, H. Oka and P. Pilarczyk. A Database Schema for the Analysis of Global Dynamics of Multiparameter Systems, SIAM J. Applied Dynamical Systems 8(2009), 757-789.

3. J-P.Aubin and A. Cellina. Differential Inclusions, Set-Valued Maps And Viability Theory, Grundl. der Math. Wiss. 264, Springer, Berlin, 1984.

4. B. Batko. Weak index pairs and the Conley index for discrete multivalued dynamical systems. Part II: properties of the Index, SIAM J. Applied Dynamical Systems 16(2017), 1587-1617.

5. B. Batko and M. Mrozek. Weak index pairs and the Conley index for discrete multivalued dynamical systems, SIAM J. Applied Dynamical Systems 15(2016), 1143-1162.

6. U. Bauer and H. Edelsbrunner. The Morse theory of Čech and Delaunay complexes, Transactions of the American Mathematical Society 369(2017), 3741-3762.

7. D. Brewer, M. Barenco, R. Callard, M. Hubank and J. Stark. Fitting ordinary differential equations to short time course data, Phil. Trans. R. Soc. A 366(2008), 519-544.

8. J. Bush, M. Gameiro, S. Harker, H. Kokubu, K. Mischaikow, I. Obayashi and P. Pilarczyk. Combinatorial-topological framework for the analysis of global dynamics, Chaos 22(2012), 047508.

9. Ch. Conley. Isolated Invariant Sets and the Morse Index, American Mathematical Society, Providence, RI, 1978.

10. J. Dydak. An addendum to the Vietoris-Begle theorem, Topology and its Applications 23(1986), 75-86.

11. Z. Dzedzej and W. Kryszewski. Conley type index applied to Hamiltonian inclusions, J. Math. Anal. Appl. 347(2008), 96-112.

12. Z. Dzedzej and G. Gabor. On homotopy Conley index for multivalued flows in Hilbert spaces, Topol. Methods Nonlinear Anal. 38(2011), 187-205.

13. D. Farley and L. Sabalka. Discrete Morse theory and graph braid groups, Algebraic and Geometric Topology 5(2005), 1075-1109.

14. R. Forman. Morse theory for cell complexes, Advances in Mathematics 134(1998), 90-145.

15. R. Forman. Combinatorial vector fields and dynamical systems, Mathematische Zeitschrift 228(1998), 629-681.

16. L. Górniewicz. Topological Fixed Point Theory of Multivalued Mappings, $2^{\text {nd }}$ ed., Topological Fixed Point Theory and Its Applications 4, Springer Verlag, The Netherlands, 2006.

17. S. Harker, K. Mischaikow, M. Mrozek and V. Nanda. Discrete Morse Theoretic Algorithms for Computing Homology of Complexes and Maps, Foundations of Computational Mathematics 14(2014) 151-184.

18. T. Kaczynski and M. Mrozek. Conley index for discrete multivalued dynamical systems, Topology and its Applications 65(1995), 83-96.

19. T. Kaczynski, M. Mrozek and T. Wanner. Towards a formal tie between combinatorial and classical vector field dynamics, J. Computational Dynamics 3(1)(2016), 17-50.

20. J. Kasten, J. Reininghaus, I. Hotz, H.C. Hege, B.R. Noack, G. Daviller, M. Morzynski. Acceleration feature points of unsteady shear flows, Archives of Mechanics 68(2016), 55-80.

21. T. Lewiner, H. Lopes and G. Tavares. Applications of Forman's discrete Morse theory to topology visualization and mesh compression, IEEE Trans. Visualiz. Comput. Graph. 10(2004), 499-508.

22. M.C. McCord. Singular homology and homotopy groups of finite spaces, Duke Math. J. 33(1966), $465-474$

23. J.W. Milnor. On the Steenrod homology theory. In: Novikov conjectures, index theorems and rigidity, Vol. 1 (Oberwolfach, 1993), London Math. Soc. Lecture Note Ser. 226(1995), Cambridge University Press, 79-96.

24. K. Mischaikow and M. Mrozek. Chaos in Lorenz equations: a computer assisted proof, Bull. AMS (N.S.) 33(1995), 66-72.

25. K. Mischaikow and V. Nanda. Morse Theory for Filtrations and Efficient Computation of Persistent Homology, Discrete and Computational Geometry 50(2013), 330-353.

26. M. Morse. The Calculus of Variations in the Large, Colloquium Publication 18, American Mathematical Society, 1934.

27. M. Mrozek. Leray functor and cohomological index for discrete dynamical systems, Trans. AMS 318(1990), 149-178.

28. M. Mrozek. A cohomological index of Conley type for multivalued admissible flows, J. Diff. Equ. 84(1990), 15-51.

29. M. Mrozek. The Conley index on compact ANR's is of finite type, Results in Mathematics 18(1990), 306-313. 
30. M. Mrozek. Normal functors and retractors in categories of endomorphisms, Univ. Iag. Acta Math. 29(1992), 181-198.

31. M. Mrozek. Shape index and other indices of Conley type for continuous maps in locally compact metric spaces, Fund. Math. 145(1994), 15-37.

32. M. Mrozek. Topological invariants, multivalued maps and computer assisted proofs in Dynamics, Computers Math. Applic. 32(1996), 83-104.

33. M. Mrozek. Conley-Morse-Forman theory for combinatorial multivector fields on Lefschetz complexes, Foundations of Computational Mathematics 17(2017), 1585-1633.

34. V. Robins, P.J. Wood, and A.P. Sheppard. Theory and algorithms for constructing discrete Morse complexes from grayscale digital images, IEEE Transactions on Pattern Analysis and Machine Intelligence 33(8)(2011), 1646-1658.

35. E. Roxin. Stability in general control systems, J. Diff. Equ. 1(1965), 115-150.

36. E. Roxin. On generalized dynamical systems defined by contigent equations, J. Diff. Equ. 1(1965), 188-205.

37. M. Schmidt and H. Lipson. Distilling Free-Form Natural Laws from Experimental Data, Science 324(2009), 81-85.

38. T. Sousbie. The persistent cosmic web and its filamentary structure I. Theory and implementation, Mon. Not. R. Astron. Soc. 414(2011), 350-383.

39. E. Spanier. Algebraic Topology, McGraw-Hill, New York, 1966.

40. K. Stolot. Homotopy Conley index for discrete multivalued dynamical systems, Topology and its Applications 153(2006), 3528-3545.

41. A. Szymczak, The Conley index for discrete semidynamical systems, Topology and its Applications 66(1995) 215-240.

42. T. Ważewski. Sur une condition équivalente à l'équation au contingent, Bull. Acad. Polon. Sci. 9(1961), $865-867$.

Publisher's Note Springer Nature remains neutral with regard to jurisdictional claims in published maps and institutional affiliations.

\section{Affiliations}

\section{Bogdan Batko ${ }^{1} \cdot$ Tomasz Kaczynski $^{2} \cdot$ Marian Mrozek $^{1} \cdot$ Thomas Wanner $^{3}$}

Bogdan Batko

Bogdan.Batko@uj.edu.pl

Tomasz Kaczynski

tomasz.kaczynski@usherbrooke.ca

Thomas Wanner

twanner@gmu.edu

1 Division of Computational Mathematics, Faculty of Mathematics and Computer Science, Jagiellonian University, ul. St. Łojasiewicza 6, 30-348 Kraków, Poland

2 Département de Mathématiques, Université de Sherbrooke, 2500 Boul. Université, Sherbrooke, QC J1K 2R1, Canada

3 Department of Mathematical Sciences, George Mason University, Fairfax, VA 22030, USA 